\title{
Plants of Genus Mentha: From Farm to Food Factory
}

\author{
Bahare Salehi ${ }^{1,2}$ (D), Zorica Stojanović-Radić ${ }^{3}$, Jelena Matejić ${ }^{4}$, Farukh Sharopov 5 (D), \\ Hubert Antolak ${ }^{6}$, Dorota Kręgiel ${ }^{6}$ (D), Surjit Sen ${ }^{7}$, Mehdi Sharifi-Rad ${ }^{8, *}$, Krishnendu Acharya ${ }^{7}$,

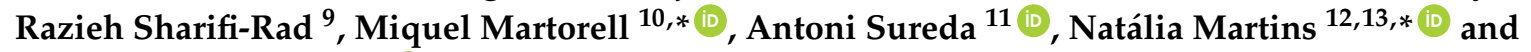 \\ Javad Sharifi-Rad 14,15,* (D)
}

1 Medical Ethics and Law Research Center, Shahid Beheshti University of Medical Sciences, Tehran 1983963113, Iran; bahar.salehi007@gmail.com

2 Student Research Committee, Shahid Beheshti University of Medical Sciences, Tehran 1983963113, Iran

3 Department of Biology and Ecology, Faculty of Sciences and Mathematics, University of Niš, Višegradska 33, 18000 Niš, Serbia; zstojanovicradic@yahoo.com

4 Department of Pharmacy, Faculty of Medicine, University of Niš, Boulevard Dr Zorana Đinđića 81, 18000 Niš, Serbia; jekamatejic@gmail.com

5 Department of Pharmaceutical Technology, Avicenna Tajik State Medical University, Rudaki 139, Dushanbe 734003, Tajikistan; shfarukh@mail.ru

6 Institute of Fermentation Technology and Microbiology, Lodz University of Technology, 90-924 Łódź, Poland; hubert.antolak@gmail.com (H.A.); dorota.kregiel@p.lodz.pl (D.K.)

7 Molecular and Applied Mycology and Plant Pathology Laboratory, Department of Botany, Centre of Advanced Study, University of Calcutta, 35, Ballygunge Circular Road, Kolkata 700019, India; surjitsen09@gmail.com (S.S.); krish_paper@yahoo.com (K.A.)

8 Department of Medical Parasitology, Zabol University of Medical Sciences, Zabol 61663335, Iran

9 Zabol Medicinal Plants Research Center, Zabol University of Medical Sciences, Zabol 61615585, Iran; razieh.sharifirad@gmail.com

10 Department of Nutrition and Dietetics, Faculty of Pharmacy, University of Concepcion, Concepcion, 4070386 VIII-Bio Bio Region, Chile

11 Research Group on Community Nutrition and Oxidative Stress and CIBEROBN (Physiopathology of Obesity and Nutrition), University of Balearic Islands, 07122 Palma de Mallorca, Spain; tosugo@hotmail.com

12 Faculty of Medicine, University of Porto, Alameda Prof. Hernâni Monteiro, 4200-319 Porto, Portugal

13 Institute for Research and Innovation in Health (i3S), University of Porto, 4200-135 Porto, Portugal

14 Phytochemistry Research Center, Shahid Beheshti University of Medical Sciences, Tehran 11369, Iran

15 Department of Chemistry, Richardson College for the Environmental Science Complex, The University of Winnipeg, Winnipeg, MB R3B 2E9, Canada

* Correspondences: mehdi_sharifirad@yahoo.com (M.S.-R.); martorellpons@gmail.com (M.M.); ncmartins@med.up.pt (N.M.); javad.sharifirad@gmail.com (J. S.-R.); Tel.: +98-543-22-51-790 (M.S.-R); +56-41-266-1671 (M.M.); +35-12-2551-2100 (N.M.); +98-21-8820-0104 (J.S.-R.)

Received: 30 July 2018; Accepted: 29 August 2018; Published: 4 September 2018

\begin{abstract}
Genus Mentha, a member of Lamiaceae family, encompasses a series of species used on an industrial scale and with a well-described and developed culture process. Extracts of this genus are traditionally used as foods and are highly valued due to the presence of significant amounts of antioxidant phenolic compounds. Many essential oil chemotypes show distinct aromatic flavor conferred by different terpene proportions. Mint extracts and their derived essential oils exert notable effects against a broad spectrum of bacteria, fungi or yeasts, tested both in vitro or in various food matrices. Their chemical compositions are well-known, which suggest and even prompt their safe use. In this review, genus Mentha plant cultivation, phytochemical analysis and even antimicrobial activity are carefully described. Also, in consideration of its natural origin, antioxidant and antimicrobial properties, a special emphasis was given to mint-derived products as an interesting alternative to artificial preservatives towards establishing a wide range of applications for shelf-life extension of food ingredients and even foodstuffs. Mentha cultivation techniques markedly influence its phytochemical composition. Both extracts and essential oils display a broad spectrum of activity,
\end{abstract}


closely related to its phytochemical composition. Therefore, industrial implementation of genus Mentha depends on its efficacy, safety and neutral taste.

Keywords: Mentha genus; essential oil; chemotypes; plant extracts; culture conditions; food preservatives; antimicrobials

\section{Introduction}

Medicinal plants represent a significant source of therapeutic remedies, being also the basis of traditional or indigenous healing systems, still widely used by the majority of populations in many countries [1,2]. Recently, the ethnopharmacological potentials of these plant matrices have received important consideration by both scientists and the pharmaceutical industry towards complementing or even replacing conventional pharmacotherapies [2-11]. Moreover, many of these plants have also been highlighted for their added-food value ability, providing a dual role, i.e., food flavor and bioactive compounds [3,12-19].

Mentha species belong to the family Lamiaceae and are widely distributed in Europe, Asia, Africa, Australia, and North America [20,21]. Plants from this genus can be found in multiple and diverse environments [20,22]. Recent data, based on morphological, cytological and genetic characteristics, have shown that genus Mentha can be classified into 42 species, 15 hybrids and hundreds of subspecies, varieties and cultivars. Indeed, mint taxonomy is highly complex and there is not always a consensus. The Mentha genus is often divided into 5 sections: Audibertia, Eriodontes, Mentha, Preslia, and Pulegium [23]. Most Mentha species are perennial, contain essential oils, and are widely cultivated as industrial crops for essential oil production. A clear definition of an essential oil is mostly based on pharmaceutical standards. Typically, they are odorous products, with a complex composition, obtained from a botanically defined raw plant material by steam or dry distillation, or even by another suitable mechanical process without heating. The term "essential oil" comes from the sense that it contains "the essence of" the plant's fragrance, not to be confused with its being essential for the functioning of plant cells or plant homeostasis. The main Mentha oil producers are USA, India and China [24]. The most economically important species are Mentha aquatica L., Mentha canadensis L., Mentha spicata L. (spearmint) and their hybrids (including Mentha $\times$ piperita L., widely known as peppermint, a sterile and first-generation hybrid between M. aquatica and M. spicata) [23]. Leaves, flowers and stems of Mentha species have traditionally been used as herbal teas and spices in many foods to add aroma and flavor [25,26]. Fresh and dried plant material, raw extracts and essential oils of mint plants are used as a part of confectionary, as flavor enhancing agents in toothpastes, chewing gums and beverages, bakery, cosmetics, as oral hygiene products, pharmaceuticals and pesticides [27]. The two most popular mint flavors are due to (-)-menthol, a terpene alcohol with strong, intense and refreshing peppermint flavor, mostly obtained from M. aquatica and M. canadensis hybrids, and $R$-(-)-carvone, a terpene ketone with a typical flavor of Maghrebi mint tea or mint cocktails, obtained from M. spicata. Another distinct flavor can be obtained from linalool-rich species, a terpene alcohol, for example $M$. arvensis L. Also, (-)-menthol, in its pure form (extracted or synthetized), can also be used in the food and cosmetic industries. Indeed, mint species have a long history of use, and are well-known for their medicinal properties [28]. Mint species, in particular their essential oils, and even menthol have been described in distinct monographs, such as European, Japanese and Russian pharmacopoeias.

Mentha leaves have traditionally been used as tea in the treatment of headache, fever, digestive disorders and various minor ailments [26]. Furthermore, mint essential oils have been widely used in the treatment of mild-intensity fungal and bacterial infections of human skin, headache syndromes and postherpetic neuralgia [27]. In modern medicine, Mentha species are widely used in the treatment of gastrointestinal tract disorders. For example, Mentha longifolia (L.) methanolic extract, rich in 
eucalyptol, showed antiulcer activity against acetic acid-induced colitis in rats, perhaps attributed to its antioxidant and anti-inflammatory effects, although the observed effect was not dose-dependent [29]. The anti-inflammatory effect of mint essential oils is being corroborated by some clinical studies; for example, M. spicata essential oil is able to reduce pain in osteoarthritis patients. This analgesic effect is mostly related to the main components of $M$. spicata essential oil, like carvone, limonene and menthol [30,31].

The interest in studying mint for human beings is majorly related to its phytosanitary effects. In fact, M. pulegium L. essential oil showed high efficiency against the food pest Sitophilus granaries, and could additionally be used as a bio-insecticide [32]. M. $\times$ piperita L. aqueous extract improved radish seedlings (Raphanus sativus L.) defensive responses against oxidative stress and had inhibitory effects on seed germination and seedling growth, thus representing a huge area of interest for natural herbicide formulation [33]. Mint phenols have also shown strong antioxidant, acetylcholinesterase (AChE), butyrylcholinesterase (BChE) and histone deacetylase inhibitory effects [34-38].

\section{Genus Mentha Plant Cultivation}

Mints are a group of perennial herbs commercially cultivated in different parts of the world. It is believed that the genus originated in the Mediterranean basin and, from there, spread to the rest of the world by both natural and artificial means. M. arvensis is cultivated on a huge scale in Brazil, China, Paraguay, Japan, Thailand, Angola and India. Peppermint is grown in the USA, Morocco, Argentina, France, Hungary, Italy and Switzerland [39]. Spearmint (M. spicata) is mainly cultivated in the USA, and bergamot mint (several hybrids rich in linalool and linalyl acetate) is native to Europe and has been naturalized in the eastern USA. Spearmint and bergamot mint are significantly less cultivated than peppermint. Currently, most of the commercially important mints are hybrids or amphiploids.

\subsection{Agro-Climatic Requirements}

M. arvensis can be cultivated both in tropical and sub-tropical areas. It does not grow well in areas with damp winters which cause root rot. A temperature of about $20-25^{\circ} \mathrm{C}$ promotes vegetative growth, but essential oil and menthol concentrations have been reported to increase at a higher temperature $\left(30^{\circ} \mathrm{C}\right)$. Peppermint and spearmint cannot be profitably grown in tropical and subtropical areas. Peppermint is grown in cool to temperate regions. It needs long days with warm to hot conditions and cool nights to ensure the right balance of essential oil compounds, produced during the growing phase. Optimum temperature is between 21 and $26^{\circ} \mathrm{C}$ for peppermint growth and flowering [40]. Bergamot mint can be grown both in temperate and sub-tropical areas. However, higher yields are observed in temperate climates, and rainfall should be around 100-110 cm. Light showers at planting time and sunny days at the harvesting stage are best for high yield and good quality of leaves.

\subsection{Soil}

Mints are not fussy about soil types and can tolerate a fairly wide range of soil chemistry and conditions. Loam and sandy loam to deep soil, rich in humus is ideal for mint cultivation [41]. The most important factors to consider are $\mathrm{pH}$ (6-7.5), organic content, overall water holding capacity and drain ability. Indeed, fresh weight, dry weight and essential oil yield of peppermint are influenced by soil $\mathrm{pH}$ [42].

\subsection{Land Preparation}

Land must be cleared with ploughing to release weeds. Mint needs thorough two cross harrowing to bring the soil to fine tilth. About 25-30 tons of farm yard manure (compost)/ha has to be applied as a basal dressing during the last ploughing to incorporate manure with soil. Green manure, like sun-hemp (Crotalaria juncea L.), should be used before plantation. Raised beds are the best and most economical way to grow mint. 


\subsection{Propagation}

Mints are perennial herbs propagated mainly by vegetative means rather than seeds. In fact, seed propagation has been practiced by some farmers, namely M. arvensis, M. pulegium and M. spicata, as these species produce viable seeds [43]. Peppermint is a popular and commercially grown hybrid mint, but is sterile, therefore its propagation exclusively depends on vegetative parts, like green shoots, underground stolons and rooty turions [44]. El-Keltawi and Croteau [45] have observed a maximum rooting percentage using etiolated rhizome, which was used as a single node cutting. It has also been estimated that 1 ha of well-grown plant material can supply a cropping area of 7 to 10 ha [46].

\subsection{Planting}

Planting materials are cut into small pieces and planted in shallow furrows about 7 to $10 \mathrm{~cm}$ deep with a row to row distance of 45-60 cm either manually or mechanically [47]. The plot needs irrigation immediately after planting, and depending on cultivation procedure, soil and climatic factors, plantation lasts about 3 to 5 years.

\subsection{Fertilization}

Exhaustive studies have been made to understand the role of nitrogen $(\mathrm{N})$, phosphorus $(\mathrm{P})$ and potassium $(\mathrm{K})$ requirements on herbage production and essential oil yield. Indeed, $\mathrm{N}$ application at 160 $\mathrm{kg} \mathrm{N} /$ ha for M. arvensis, $125 \mathrm{~kg} \mathrm{~N} /$ ha for M. $\times$ piperita, $100-120 \mathrm{~kg} \mathrm{~N} /$ ha for M. aquatica and M. spicata give a higher dry matter amount and essential oil yield [48-50]. Helsel and Fluck [51] have shown a correlation between $\mathrm{N}$ fertilizer application and (-)-carvone and limonene concentrations in Mentha $\times$ gentilis L. Generally, 80-120 kg N fertilizer, $50 \mathrm{~kg} \mathrm{P}_{2} \mathrm{O}_{5}$ and $40 \mathrm{~kg} \mathrm{~K}_{2} \mathrm{O} / \mathrm{ha}$ is required for a good mint crop. In fact, experiments conducted at the Central Institute of Medicinal and Aromatic Plants (CIMAP, Lucknow, India) have shown that the application of $20 \mathrm{~kg} /$ ha sulphur (S) increase M. spicata foliage and essential oil yield [46].

\subsection{Irrigation}

Mint requires frequent and adequate irrigation. Fully developed plants are watered at least three times a week. It is important to keep the soil constantly moist, although well drained. The crop has high water demands in summer. Care has to be taken to prevent a waterlogged soil, especially in winter, as this will influence growth [52].

Several studies have shown that peppermint and spearmint biomass production is highly sensitive to water stress [49,53-56]. Therefore, to increase peppermint yield, furrow irrigation is preferred to sprinkler irrigation, which damages leaf oil cells gland and reduces marketable oil yield [57]. Mitchell and Yang [54] reported that alternate furrow irrigation method is more advantageous over conventional furrow irrigation, as it allows frequent application of small water amounts.

\subsection{Effect of Cultivation Conditions on Essential Oil Content}

Environmental stress can negatively affect the quality of peppermint essential oil. Depending on the edaphoclimatic conditions, the content of phenolics and terpenes can be different. For example, the concentration of menthol in peppermint is higher in flowering stage than during budding period [58]. Research on biotechnological methods for mint oil production has gained pivotal attention due to its economic prospects. Several strategies were attempted for cloning and characterizing key genes involved in mint oil components biosynthesis [59]. DNA library creation encoding oil gland secretory cells and the collection of sequence tag expression provide information on gene expression and metabolic regulation of essential oil biosynthesis [60]. 


\subsection{Pests and Diseases}

Like other cultivated medicinal plants, mints are also susceptible to exposure to a wide variety of pests and diseases, causing significant crop loss and deterioration of mint oils quality [61]. Different causal organisms, including fungi [61-68], insects [46], nematodes [69,70], bacteria [71] virus and phytoplasma [72,73], are among to the common threats on mint cultivation. Among these fungi, Puccinia menthae (rust), Alternaria alternata (leaf spot); Verticillium dahliae (wilt), Phoma stasserti (stem rot), Rhizoctonia solani (root and stolon rot) and Erysiphe cichoracearum (powdery mildew) cause serious economic loss worldwide. In fact, diseases control through chemicals appears to be more attractive, though alternative strategies like botanicals and biopesticides are also practiced to prevent mint diseases $[61,64,74]$. Additionally, mint diseases control can also be achieved through breeding programs that develop resistant clones [75].

\subsection{Harvesting}

Mint harvesting must take place at the proper time, since harvesting timings that are too early or too late result in immature or over-mature crops, which ultimately gives poor essential oil quality and quantity [76]. Another important factor is the number of harvests per year, which also influences forage yield and essential oil composition [77]. The crop must be harvested when plants are in full bloom, in the late morning on a dry sunny day, when all dew traces have disappeared. According to climatic conditions, one or two crop flushes are harvested. In addition, mint essential oil quality differs between first and second harvest, as does the oil content between the first and second year of $M$. arvensis harvest [78]. In brief, the plant gives the best crop in second and third year after planting $[79,80]$.

\section{Phytochemical Composition of Essential Oils Obtained from Genus Mentha}

\subsection{Volatile Compounds}

Essential oils are usually detached from aqueous phase through a physical process that does not significantly affect their composition. Characteristically appearing in their liquid, volatile, limpid and rarely colored form, essential oils also display a good solubility in lipids and organic solvents, often having lower densities than that of water [81]. Odorous secondary metabolite biosynthesis in Mentha species occurs in peltate glandular trichomes, specialized epidermal tissues located on leaves, stems, petals and seed coat surfaces, depending on the species [59,60].

Mint plants either produce C3-oxygenated $p$-menthane (e.g., pulegone, menthone, menthol) or C6-oxygenated $p$-menthane (e.g., carvone) types, as major monoterpenes [82]. Hydrocarbons ( $\beta$-caryophyllene, germacrene $\mathrm{D}$, limonene), alcohols (elemol, geraniol, linalool, menthol, neomenthol, 3-octanol, cis/trans-sabinene hydrate, $\alpha$-terpineol, terpinen-4-ol, viridoflorol), esters (decyl acetate, dihydrocarvyl acetate, 1,2-epoxyneomenthyl acetate, menthyl acetate, neoisomenthyl acetate, neomenthyl acetate, 3-octyl acetate, $\alpha$-terpinyl acetate), ketones (carvone, cis-/trans- $d$ ihydrocarvone, isomenthone, menthone, 3-octanone, pulegone, piperitenone, piperitone), and ethers (1,8-cineole, menthofuran, caryophyllene oxide, piperitenone oxide, piperitone oxide) were also found as main components of Mentha species essential oils (Figure 1) [83].

Members of the genus Mentha show a great variability in chemical composition, both intraand inter-species. Reports on chemical composition of various Mentha species showed that many factors interfere with essential oil composition, among them environmental (growth location, soil characteristics, moisture presence, temperature, etc.), phenological (phase of the plant collection), plant part used for essential oil extraction (flowers, stems, leaves, entire aerial parts or inflorescences), type of material (fresh or dry), and even methods used for essential oil analysis (Table 1).

Some Mentha species present numerous chemotypes, and the distinct chemical patterns are a consequence of their specific metabolic pathways, e.g., linalool, menthol or (-)-carvone pathway [84], and of their intraspecific chemical differences, e.g., chemotypes [85]. So far, the highest number of chemical constituents $(n=82)$ was reported to M. longifolia collected in Tajikistan [86]. On the other 
hand, only three compounds have been identified in essential oil isolated from M. spicata leaves grown in Brazil [87]. Indeed, such data markedly depend on the sensitivity of the analytical method used, besides handling, harvesting time and growth conditions.

There have been numerous reports on M. aquatica essential oil composition, in which menthofuran was the main compound identified in the largest number of oils analyzed, ranging from $11-70.5 \%$ of the total oil content [88-95]. Menthofuran is followed by 1,8-cineole, $\beta$-caryophyllene, limonene and viridiflorol, recorded as the most dominant compounds in M. aquatica essential oil. It has been reported that $M$. aquatica possess 26 essential oil chemotypes isolated from aerial parts, leaves, inflorescences, seeds or stolons [95]. In addition to the aforementioned constituents, linalyl acetate, piperitenone oxide, pulegone, menthone, elemol, caryophyllene oxide, $\alpha$-terpinene, isopinocamphone, linalool, menthol, menthyl acetate, germacrene $\mathrm{D}$, dihydrocarveil acetate, menthyl esters and $\alpha$-pinene are also found in M. aquatica essential oil [95].<smiles>C=C1CC=C(C)CCC1C(C)C</smiles><smiles>C=C(/C=C/CC(C)C)CCC(C)C</smiles><smiles>C=CC1CCC(C(C)(C)O)CC1C(C)=C(C)C</smiles><smiles>C=CC(O)(Br)CC=C(C)C</smiles><smiles>CC(C)C(C)CCCCCC(O)CCCCCC1CCCCC1C(C)C(C)C</smiles><smiles>CC1CCC(C)(C)C2(C)CCCCC12O</smiles><smiles>[Te][Te]</smiles>

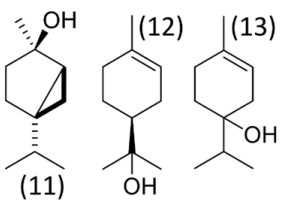

(11)<smiles>C=C(C)C1CCC(C)C(C(=C)C)C1</smiles><smiles>CC1CCC(C(C)C)C(OC(=O)CC23CC(C)CCC2(C(C)C)O3)C1</smiles><smiles>CCCCCCCCCCOC(C)=O</smiles><smiles>CC(=O)OC1CCCC(C)C1</smiles><smiles>CC1CCC(C)C(OC(=O)CC(=O)OC2CC(C)CCC2C(C)C(C)C)C1</smiles><smiles>CCCCCC1CCCCC1OC(C)=O</smiles><smiles>CCC(=O)OC(C)(C)C1CC=C(C)CC1</smiles><smiles>C=C(C)C1CC=C(C)C(=O)C1</smiles>

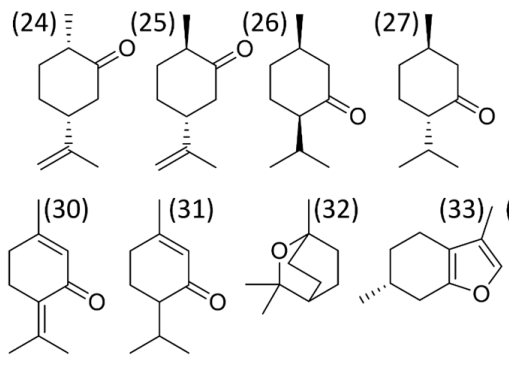

(28)

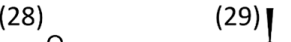

(29)

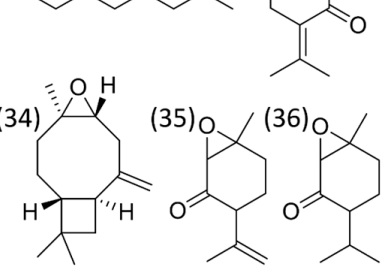

Figure 1. Main components of Mentha species essential oils: (1) $\beta$-caryophyllene, (2) germacrene $\mathrm{D}$, (3) limonene, (4) elemol, (5) geraniol, (6) linalool, (7) menthol, (8) neomenthol, (9) 3-octanol, (10) cis-sabinene hydrate, (11) trans-sabinene hydrate, (12) $\alpha$-terpineol, (13) terpinen-4-ol, (14) viridoflorol, (15) decyl acetate, (16) dihydrocarvyl acetate, (17) 1,2-epoxyneomenthyl acetate, (18) menthyl acetate, (19) neoisomenthyl acetate, (20) neomenthyl acetate, (21) 3-octyl acetate, (22) $\alpha$-terpinyl acetate, (23) carvone, (24) cis-dihydrocarvone, (25) trans-dihydrocarvone, (26) isomenthone, (27) menthone, (28) 3-octanone, (29) pulegone, (30) piperitenone, (31) piperitone, (32) 1,8-cineole, (33) menthofuran, (34) caryophyllene oxide, (35) piperitenone oxide, and (36) piperitone oxide. 
Table 1. Main findings on phytochemical analysis of Mentha species.

\begin{tabular}{|c|c|c|c|c|c|c|c|c|}
\hline \multicolumn{3}{|c|}{ Plant } & \multicolumn{3}{|c|}{ Extraction } & \multicolumn{2}{|r|}{ Compounds } & \multirow{2}{*}{ References } \\
\hline Part & Material & Origin & Method & Identification & Yield & Number & Most Abundant & \\
\hline \multicolumn{9}{|c|}{ Mentha aquatica $\mathrm{L}$. } \\
\hline ns & Fresh & Romania & HD & GC-MS & ns & 41 & $\begin{array}{l}\text { menthofuran }(58.59 \%) \text {, } \\
\text { limonene }(9.91 \%) \\
\text { trans- } \beta \text {-ocimene }(5.59 \%) \\
\text { ledol }(3.29 \%) \\
\beta \text {-caryophyllene }(3.55 \%)\end{array}$ & [94] \\
\hline $\mathrm{AP}$ & Dried & Vietnam & SD & GC and GC-MS & $\begin{array}{l}0.42 \% \text { (TH) } \\
0.34 \% \text { (NA) }\end{array}$ & 28 & $\begin{array}{l}\text { Thanh Hóa province: } \\
\text {-epi-bicyclosesquiphellandrene (58.9\%) } \\
\text {-limonene (21.1\%) } \\
\text { Nghệ an province: } \\
\text {-epi-bicyclosesquiphellandrene (52.4\%) } \\
\text {-limonene (31.4\%) }\end{array}$ & [95] \\
\hline $\mathrm{AP}$ & ns & Brazil & HD & GC-MS & $0.26 \%$ & 19 & $\begin{array}{l}\text { D-carvone }(58.79 \%) \\
\text { limonene }(28.29 \%)\end{array}$ & [96] \\
\hline \multicolumn{9}{|c|}{ Mentha arvensis $\mathrm{L}$. } \\
\hline $\mathrm{L}$ & Fresh & Brazil & DHM & GC-MS & ns & 7 & $\begin{array}{l}\text { Young leaves: } \\
\text {-menthol }(81.46 \%) \\
\text {-pulegone }(7.25 \%) \\
\text {-p-cymene }(3.27 \%) \\
\text { Mature leaves: } \\
\text {-menthol }(86.87 \%) \\
\text {-p-cymene }(4.38 \%) \\
\text {-pulegone }(3.47 \%)\end{array}$ & [97] \\
\hline ns & ns & Brazil & SD & GC-MS & ns & 26 & $\begin{array}{l}\text { menthol }(56.85 \%) \\
\text { isomenthone }(21.13 \%) \\
\text { menthyl acetate }(4.62 \%) \\
\text { limonene }(4.07 \%) \\
\text { isopulegol }(3.71 \%)\end{array}$ & [83] \\
\hline $\mathrm{AP}$ & - & Brazil & $\mathrm{HD}$ & GC-MS & $0.10 \%$ & 21 & $\begin{array}{l}\text { linalyl acetate }(39.72 \%) \\
\text { linalool }(34.57) \\
\text { 1.8-cineole }(10.04 \%)\end{array}$ & [96] \\
\hline \multicolumn{9}{|c|}{ Mentha canadensis $\mathrm{L}$. } \\
\hline $\mathrm{AP}$ & Fresh & China & HD & $\begin{array}{c}\text { GC } \\
\text { GC-MS }\end{array}$ & $0.02-0.18 \%$ & 16 & $\begin{array}{l}\text { menthol }(80.47 \%) \\
\text { menthone }(7.25 \%) \\
\text { isomenthone }(2.31 \%) \\
\text { isopulegol }(2.17 \%) \\
\text { pulegone }(2.05)\end{array}$ & [98] \\
\hline
\end{tabular}


Table 1. Cont

\begin{tabular}{|c|c|c|c|c|c|c|c|c|}
\hline \multicolumn{3}{|c|}{ Plant } & \multicolumn{3}{|c|}{ Extraction } & \multicolumn{2}{|r|}{ Compounds } & \multirow{2}{*}{ References } \\
\hline Part & Material & Origin & Method & Identification & Yield & Number & Most Abundant & \\
\hline $\mathrm{AP}$ & ns & China & HD & GC and GC-MS & $1.42 \%$ & 36 & $\begin{array}{l}\text { menthol }(28.8 \%) \\
\alpha \text {-pinene }(16.4 \%) \\
\text { menthone }(12.7 \%) \\
\alpha \text {-terpineol }(6.3 \%) \\
\text { limonene }(5.5 \%)\end{array}$ & [99] \\
\hline AP & ns & Brazil & HD & GC-MS & $0.54 \%$ & 24 & $\begin{array}{l}\text { menthol (46.98\%) } \\
\text { isomenthone (29.07\%) }\end{array}$ & [96] \\
\hline \multicolumn{9}{|c|}{ Mentha cardiaca J. Gerard ex Baker } \\
\hline $\mathrm{AP}$ & ns & India & HD & GC-MS & $12 \mathrm{~mL} / \mathrm{kg}$ & 60 & $\begin{array}{l}\text { carvone }(59.6 \%) \\
\text { limonene }(23.3 \%) \\
\beta \text {-myrcene }(2.5 \%) \\
\text { 1,8-cineole }(2.1 \%) \\
\text { cis-dihydrocarvone }(1.5 \%) \\
\beta \text {-Bourbonene }(1.5 \%)\end{array}$ & [100] \\
\hline \multicolumn{9}{|c|}{ Mentha cervina $\mathrm{L}$. } \\
\hline $\mathrm{AP}$ & Dried & Portugal & HD & $\begin{array}{c}\text { GC } \\
\text { GC-MS }\end{array}$ & ns & 28 & $\begin{array}{l}\text { August (flowering phase): } \\
\text {-pulegone }(75.1 \%), \text { isomenthone }(8.7 \%) \\
\text {-limonene }(4.3 \%) \\
\text { Vegetative phases: } \\
\text {-October: pulegone } 79.6 \% \text {, isomenthone } \\
9.6 \% \text {, limonene }(3.2 \%) \\
\text {-December: pulegone } 58.3 \% \text {, isomenthone } \\
33.3 \% \text {, limonene } 1.2 \% \\
\text {-February: pulegone } 12.9 \% \text {, isomenthone } \\
77.0 \% \text {, menthon } 4.4 \%\end{array}$ & [101] \\
\hline $\mathrm{AP}$ & ns & Portugal & HD & $\begin{array}{c}\text { GC } \\
\text { GC-MS }\end{array}$ & ns & 33 & $\begin{array}{l}\text { pulegone }(52-75 \%) \\
\text { isomenthone }(8-24 \%) \\
\text { limonene }(4-6 \%) \\
\text { menthone }(1-2 \%)\end{array}$ & [102] \\
\hline $\mathrm{AP}$ & ns & Portugal & HD & $\begin{array}{c}\text { GC } \\
\text { GC-MS }\end{array}$ & $2.4-4.0 \%$ & 25 & $\begin{array}{l}\text { cultivated populations: } \\
\text { pulegone }(62-78 \%) \\
\text { isomenthone }(3.1-15 \%) \\
\text { limonene }(3.4-7.4 \%) \\
\text { wild populations. } \\
\text { pulegone }(73-80 \%) \\
\text { isomenthone } \\
(6.1-18.2 \%) \\
\text { limonene }(4.5-5.2 \%)\end{array}$ & [85] \\
\hline
\end{tabular}


Table 1. Cont

\begin{tabular}{|c|c|c|c|c|c|c|c|c|}
\hline \multicolumn{3}{|c|}{ Plant } & \multicolumn{3}{|c|}{ Extraction } & \multicolumn{2}{|r|}{ Compounds } & \multirow{2}{*}{ Reference } \\
\hline Part & Material & Origin & Method & Identification & Yield & Number & Most Abundant & \\
\hline \multicolumn{9}{|c|}{ Mentha diemenica Spreng. } \\
\hline $\mathrm{L}$ & ns & $\begin{array}{c}\text { Canada } \\
\text { Australia }\end{array}$ & SD & $\begin{array}{c}\text { GC } \\
\text { GC-MS }\end{array}$ & $1.0 \%$ & 35 & $\begin{array}{l}\text { Australia: } \\
\text {-menthone }(32.4 \%) \\
\text {-pulegone }(24.9 \%) \\
\text {-neomenthyl acetate }(18.3 \%) \\
\text {-neomenthol }(9.0 \%) \\
\text {-menthyl acetate }(5.7 \%) \\
\text {-menthol }(1.8 \%) \\
\text {-isomenthone }(1.0 \%) \\
\text { Canada: } \\
\text {-pulegone }(43.6 \%) \\
\text {-menthone }(32.2 \%) \\
\text {-isomenthone }(3.2 \%) \\
\text {-menthyl acetate }(2.7 \%) \\
\text {-neomenthol }(2.5 \%) \\
\text {-menthol }(2.7 \%)\end{array}$ & [103] \\
\hline \multicolumn{9}{|c|}{ Mentha longifolia $\mathrm{L}$. } \\
\hline $\mathrm{L}$ & Air-dried & Turkey & HD & GC-MS & ns & 40 & $\begin{array}{l}\text { menhone }(19.31 \%) \\
\text { pulegone }(12.42 \%) \\
\text { piperitone }(11.05 \%) \\
\text { dihydrocarvon }(8.32 \%) \\
\text { limonene }(6.1 \%) \\
\text { 3-terpinolenone }(5.66 \%) \\
\text { 1,8-cineole }(4.37 \%) \\
\text { germacrene D }(3.38 \%) \\
\text { caryopyllene }(3.19 \%)\end{array}$ & [104] \\
\hline $\mathrm{L}$ & Dried & Iran & HD & GC-MS & $1.34 \%$ & 24 & $\begin{array}{l}\text { Leaf oil: } \\
\text {-1,8-cineole }(37.16 \%) \\
\text {-piperitenone oxide }(18.97 \%) \\
\text {-sabinene }(13.94 \%) \\
\text { - } \alpha \text {-pinene }(8.92 \%) \\
\text {-pulegone }(6.14 \%)\end{array}$ & \multirow[t]{2}{*}{ [105] } \\
\hline$S$ & Dried & Iran & HD & GC-MS & $0.76 \%$ & 27 & $\begin{array}{l}\text { Stem oil: } \\
\text {-1,8-cineole }(36.81 \%) \\
\text {-pulegone }(18.61 \%) \\
\text {-piperitenone oxide }(12.21 \%) \\
\text {-sabinene }(7.05 \%)\end{array}$ & \\
\hline
\end{tabular}


Table 1. Cont.

\begin{tabular}{|c|c|c|c|c|c|c|c|c|}
\hline \multicolumn{3}{|c|}{ Plant } & \multicolumn{3}{|c|}{ Extraction } & \multicolumn{2}{|r|}{ Compounds } & \multirow{2}{*}{ References } \\
\hline Part & Material & Origin & Method & Identification & Yield & Number & Most Abundant & \\
\hline $\mathrm{F}$ & Dried & Iran & HD & GC-MS & $0.97 \%$ & 25 & $\begin{array}{l}\text { Flower oil: } \\
\text {-piperitenone oxide }(37.67 \%) \\
\text {-1,8-cineole }(23.02 \%) \\
\text {-sabinene }(13.56 \%) \\
-\alpha \text {-pinene }(10.45 \%)\end{array}$ & \\
\hline $\mathrm{L}$ & Fresh & $\begin{array}{l}\text { Saudi } \\
\text { Arabia }\end{array}$ & HD & GC-MS & $0.5-0.9 \%$ & 49 & $\begin{array}{l}\text { pulegone }(11.92-62.54 \%) \\
\text { menthone }(7.84-34.13 \%) \\
\text { eucalyptol }(5.96-20.07 \%)\end{array}$ & [106] \\
\hline $\mathrm{AP}$ & Dried & $\begin{array}{c}\text { Bosnia and } \\
\text { Herzegovina }\end{array}$ & HD & GC-MS & $1.9 \%$ & 36 & $\begin{array}{l}\text { piperitone oxide }(63.58 \%) \\
1.8 \text {-cineole }(12.03 \%) \\
\text { caryophyllene oxide }(4.33 \%) \\
\text { trans-caryophyllene }(2.98 \%) \\
\text { cis-caryophyllene }(0.82 \%)\end{array}$ & [107] \\
\hline $\mathrm{AP}$ & Dried & Tajikistan & HD & GC-MS & $0.5-0.9 \%$ & 82 & $\begin{array}{l}\text { cis-piperitone epoxide }(7.8-77.6 \%) \\
\text { piperitenone oxide }(1.5-49.1 \%) \\
\text { carvone }(0.0-21.5 \%) \\
\text { menthone }(0.0-16.6 \%) \\
\text { thymol }(1.5-4.2 \%) \\
\text { pulegone }(0.3-5.4 \%) \\
\beta \text {-thujone }(0.2-3.2 \%) \\
(E) \text {-caryophyllene }(0.9-2.5 \%) \\
\text { myrcene }(0.3-2.5 \%) \\
\text { carvacrol }(0.0-2.7 \%) \\
\text { borneol }(0.9-1.8 \%) \\
p \text {-cymene } \\
(0.2-1.9 \%)\end{array}$ & [86] \\
\hline $\mathrm{L}$ & Fresh & Tunisia & HD & GC-MS & $1.3 \%$ & 35 & $\begin{array}{l}\text { pulegone }(54.41 \%) \\
\text { isomenthone }(12.02 \%) \\
1,8 \text {-cineole }(7.41 \%) \\
\text { borneol }(6.85 \%) \\
\text { piperitenone oxide }(3.19 \%)\end{array}$ & [108] \\
\hline $\mathrm{AP}$ & ns & Brazil & HD & GC-MS & $0.05 \%$ & 11 & $\begin{array}{l}\text { piperitenone oxide }(60.79 \%) \\
\text { L-limonene }(13.81 \%) \\
\text { carvone }(5.21 \%) \\
\text { myrcene }(2.51 \%) \\
\beta \text {-pinene }(1.92)\end{array}$ & [96] \\
\hline
\end{tabular}


Table 1. Cont

\begin{tabular}{|c|c|c|c|c|c|c|c|c|}
\hline \multicolumn{3}{|c|}{ Plant } & \multicolumn{3}{|c|}{ Extraction } & \multicolumn{2}{|r|}{ Compounds } & \multirow{2}{*}{ References } \\
\hline Part & Material & Origin & Method & Identification & Yield & Number & Most Abundant & \\
\hline \multicolumn{9}{|c|}{ Mentha pulegium L. } \\
\hline $\mathrm{AP}$ & Dried & Algeria & HD & $\begin{array}{c}\text { GC } \\
\text { GC-MS }\end{array}$ & $2.34 \%$ & 37 & $\begin{array}{l}\text { pulegone }(46.31 \%) \\
\text { piperitenone }(23.3 \%) \\
\text { menthone }(6.2 \%) \\
\text { limonene }(4.7 \%)\end{array}$ & [109] \\
\hline $\mathrm{AP}$ & Dried & Morocco & HD & GC-MS & $5.4 \%$ & 21 & $\begin{array}{l}\text { pulegone }(40.98 \%) \\
\text { menthone }(21.164 \%) \\
\text { humulene }(5.4 \%) \\
\text { eucalyptol }(5.2 \%)\end{array}$ & [110] \\
\hline $\mathrm{AP}$ & Dried & Morocco & HD & $\begin{array}{c}\text { GC } \\
\text { GC-MS }\end{array}$ & ns & 10 & $\begin{array}{l}\text { pulegone }(73.33 \%) \\
\text { menthone }(8.63 \%) \\
\alpha \text {-pinene }(1.70 \%)\end{array}$ & [111] \\
\hline $\mathrm{AP}$ & Dried & Uruguay & HD & $\begin{array}{c}\text { GC } \\
\text { GC-MS }\end{array}$ & $1.93 \%$ & 22 & $\begin{array}{l}\text { pulegone }(73.4 \%) \\
\text { isomenthone }(12.9 \%) \\
\text { menthone }(3.6 \%)\end{array}$ & [112] \\
\hline $\mathrm{AP}$ & Dried & Algeria & HD & $\begin{array}{c}\text { GC } \\
\text { GC-MS }\end{array}$ & $1.0 \%$ & 43 & $\begin{array}{l}\text { pulegone }(38.81 \%) \\
\text { menthone }(19.24 \%) \\
\text { piperitenone }(16.52 \%) \\
\text { piperitone }(6.34 \%) \\
\text { isomenthone }(6.09 \%) \\
\text { limonene }(4.29 \%) \\
\text { octanol }(1.85 \%)\end{array}$ & [113] \\
\hline $\mathrm{AP}$ & Dried & Morocco & HD & GC-MS & $5.4 \%$ & 21 & $\begin{array}{l}\text { pulegone }(40.98 \%) \\
\text { menthone }(21.164 \%)\end{array}$ & [110] \\
\hline $\mathrm{AP}$ & Dried & Portugal & HD & GC-MS & $0.90 \%$ & 53 & $\begin{array}{l}\text { menthone }(35.9 \%) \\
\text { pulegone }(23.2 \%) \\
\text { neo-menthol }(9.2 \%)\end{array}$ & [114] \\
\hline $\mathrm{L}$ & Dried & Algeria & SD & GC-MS & $1.45 \%$ & 39 & $\begin{array}{l}\text { pulegone }(70.66 \%) \\
\text { neo-menthol }(11.21 \%) \\
\text { menthone }(2.63 \%) \\
\text { cis-isopulegone }(2.33 \%) \\
\text { piperitenone } 1.58 \%\end{array}$ & [32] \\
\hline $\mathrm{AP}$ & Dried & Morocco & SD & $\begin{array}{c}\text { GC } \\
\text { GC-MS }\end{array}$ & $2.0 \%$ & 29 & $\begin{array}{l}\text { pulegone }(73.0 \%) \\
\text { isomentone }(13.4 \%) \\
\text { menthone }(2.6 \%) \\
\text { limonene }(1.4 \%)\end{array}$ & [115] \\
\hline
\end{tabular}


Table 1. Cont

\begin{tabular}{|c|c|c|c|c|c|c|c|c|}
\hline \multicolumn{3}{|c|}{ Plant } & \multicolumn{3}{|c|}{ Extraction } & \multicolumn{2}{|r|}{ Compounds } & \multirow{2}{*}{ References } \\
\hline Part & Material & Origin & Method & Identification & Yield & Number & Most Abundant & \\
\hline \multicolumn{9}{|c|}{ Mentha spicata $\mathrm{L}$. } \\
\hline $\mathrm{L}$ & Fresh & India & HD & $\begin{array}{c}\text { GC } \\
\text { GC-MS }\end{array}$ & $6.5 \mathrm{~mL} / \mathrm{kg}$ & 18 & $\begin{array}{l}\text { carvone }(48.60 \%) \\
\text { cis-carveol }(21.30 \%) \\
\text { limonene }(11.30 \%)\end{array}$ & [116] \\
\hline $\mathrm{L}$ & ns & Brazil & HD & GC-MS & & 25 & $\begin{array}{l}\text { piperitone }(81.18 \%) \\
\text { piperitenone }(14.57 \%) \\
\text { limonene }(1.47 \%)\end{array}$ & [117] \\
\hline $\mathrm{AP}$ & Dried & Tunisia & HD & GC/MS & & 34 & $\begin{array}{l}\text { carvone }(40.8 \%) \\
\text { limonene }(20.8 \%) \text {, } \\
\text { 1,8-cineole }(17.0 \%) \\
\beta \text {-pinene }(2.2 \%) \\
\text { cis-dihydrocarvone }(1.9 \%) \\
\text { dihydrocarveol }(1.7 \%) .\end{array}$ & [118] \\
\hline $\mathrm{L}$ & Fresh & Tunisia & HD & GC-MS & $0.8 \%$ & 49 & $\begin{array}{l}\text { carvone }(50.47 \%) \\
1,8 \text {-cineole }(9.14 \%) \\
\text { limonene }(4.87 \%) \\
\text { camphor }(3.68 \%) \\
\beta \text {-caryophyllene }(3.0 \%)\end{array}$ & [108] \\
\hline $\mathrm{AP}$ & Dried & Algeria & HD & $\begin{array}{c}\text { GC } \\
\text { GC-MS }\end{array}$ & $0.87 \%$ & 57 & $\begin{array}{l}\text { carvone }(59.40 \%) \\
\text { limonene }(6.12 \%) \\
\text { 1,8-cineole }(3.80 \%) \\
\text { germacrene } \mathrm{D}(4.66 \%) \\
\beta \text {-caryophyllene }(2.96 \%) \\
\beta \text {-bourbonene }(2.79 \%) \\
\alpha \text {-terpineol }(1.98 \%) \\
\text { terpinene-4-ol }(1.12 \%) \\
\end{array}$ & [113] \\
\hline $\mathrm{AP}$ & Fresh & Senegal & HD & $\begin{array}{c}\text { GC } \\
\text { GC-MS }\end{array}$ & $0.10 \%$ & 30 & $\begin{array}{l}\text { carvone }(67.8 \%) \\
\text { limonene }(18.1 \%) \\
\text { cis-dihydrocarvone }(1.9 \%) \\
\text { trans-carveol }(2.9 \%) \\
(E)-\beta \text {-caryophyllene }(1.1 \%) \\
\text { germacrene D }(1.1 \%)\end{array}$ & [119] \\
\hline $\mathrm{AP}$ & Dried & Senegal & HD & $\begin{array}{c}\text { GC } \\
\text { GC-MS }\end{array}$ & $0.19 \%$ & 34 & $\begin{array}{l}\text { carvone }(74.7 \%) \\
\text { limonene }(12.5 \%) \\
\text { cis-dihydrocarvone }(2.0 \%) \\
\text { trans-carveol }(2.2 \%) \\
(E)-\beta \text {-caryophyllene }(1.1 \%) \\
\text { germacrene D }(1.0 \%)\end{array}$ & [119] \\
\hline
\end{tabular}


Table 1. Cont

\begin{tabular}{|c|c|c|c|c|c|c|c|c|}
\hline \multicolumn{3}{|c|}{ Plant } & \multicolumn{3}{|c|}{ Extraction } & \multicolumn{2}{|r|}{ Compounds } & \multirow{2}{*}{ References } \\
\hline Part & Material & Origin & Method & Identification & Yield & Number & Most Abundant & \\
\hline $\mathrm{AP}$ & Dried & Serbia & HD & GC-MS & ns & 27 & $\begin{array}{l}\text { carvone }(49.5 \%) \\
\text { menthone }(21.9 \%) \\
\text { limonene }(5.8 \%) \\
\text { 1,8-cineole }(3.0 \%)\end{array}$ & [120] \\
\hline $\mathrm{AP}$ & Fresh & Brazil & SD & GC & $0.32 \%$ & 3 & $\begin{array}{l}\text { piperitone oxide }(94.8 \%) \\
\gamma \text {-muurolene }(1.06 \%) \\
\beta \text {-farnesene }(0.76 \%)\end{array}$ & [87] \\
\hline $\mathrm{AP}$ & Dried & Greece & HD & $\begin{array}{c}\text { GC } \\
\text { GC-MS }\end{array}$ & $0.2 \%$ & 39 & $\begin{array}{l}\text { piperitenone oxide }(35.7 \%) \\
\text { 1,8-cineole }(14.5 \%) \\
\text { trans-calamene }(6.4 \%) \\
\text { spathulenol }(5.2 \%)\end{array}$ & [121] \\
\hline $\mathrm{AP}$ & Fresh & India & HD & $\begin{array}{l}\text { GC/FID } \\
\text { GC-MS }\end{array}$ & $0.56 \%$ & 20 & $\begin{array}{l}\text { carvone }(49.62 \text { to } 76.65 \%) \\
\text { limonene }(9.57 \text { to } 22.31 \%) \\
\text { 1,8-cineole }(1.32 \text { to } 2.62 \%) \\
\text { trans-carveol }(0.3 \text { to } 1.52 \%)\end{array}$ & [122] \\
\hline $\mathrm{AP}$ & Dried & Morocco & SD & $\begin{array}{c}\text { GC } \\
\text { GC-MS }\end{array}$ & $0.70 \%$ & 43 & $\begin{array}{l}\text { carvone }(42.3 \%) \\
\text { limonene }(11.0 \%) \\
\text { menthone }(7.2 \%)\end{array}$ & [115] \\
\hline \multicolumn{9}{|c|}{ Mentha spicata subsp. condensata (Briq.) Greuter \& Burdet } \\
\hline $\mathrm{AP}$ & Fresh & Italy & HD & $\begin{array}{c}\text { GC } \\
\text { GC-MS }\end{array}$ & $0.21 \%$ & 29 & $\begin{array}{l}\text { pulegone }(34.1 \%) \\
\text { piperitenone oxide }(32.9 \%) \\
\text { piperitenone }(11.3 \%) \\
\text { (Z)-ocimene }(3.9 \%) \\
\text { limonene }(3.9 \%) \\
\text { linalool }(2.7 \%)\end{array}$ & [123] \\
\hline \multicolumn{9}{|c|}{ Mentha suaveolens Ehrh. } \\
\hline $\mathrm{AP}$ & Dried & Morocco & HD & $\begin{array}{c}\text { GC } \\
\text { GC-MS }\end{array}$ & ns & 15 & $\begin{array}{l}\text { piperitenone }(33.03 \%) \\
\text { pulegone }(17.61 \%) \\
\text { piperitone }(9.18 \%)\end{array}$ & [111] \\
\hline $\mathrm{F}$ & Fresh & Egypt & HD & $\begin{array}{c}\text { GC } \\
\text { GC-MS }\end{array}$ & $1.7 \%$ & 29 & $\begin{array}{l}\text { carvone }(50.59 \%) \\
\text { limonene }(31.25 \%) \\
\text { trans- } \beta \text {-caryophyllene }(2.56 \%)\end{array}$ & [84] \\
\hline
\end{tabular}


Table 1. Cont

\begin{tabular}{|c|c|c|c|c|c|c|c|c|}
\hline \multicolumn{3}{|c|}{ Plant } & \multicolumn{3}{|c|}{ Extraction } & \multicolumn{2}{|r|}{ Compounds } & \multirow{2}{*}{ References } \\
\hline Part & Material & Origin & Method & Identification & Yield & Number & Most Abundant & \\
\hline $\mathrm{AP}$ & Fresh & Egypt & HD & GC-MS & $0.47-0.60 \%$ & 46 & $\begin{array}{l}\text { Spring, summer and autumn samples: } \\
\text {-carvone }(31-56 \%) \\
\text {-limonene }(22.59-29.18 \%) \\
\text { Winter samples: } \\
\text {-limonene }(26 \%) \\
\text {-carvone }(25 \%)\end{array}$ & [124] \\
\hline $\mathrm{AP}$ & & Morocco & SD & $\begin{array}{l}\text { IR, NMR and } \\
\text { MS }\end{array}$ & $0.012 \%$ & 31 & $\begin{array}{l}\text { pulegone }(0.1-50 \%) \\
\text { piperitenone oxide }(0.9-56 \%) \\
\text { piperitone oxide }(0.3-26 \%)\end{array}$ & [125] \\
\hline $\mathrm{L}$ & Dried & China & HD & GC-MS & $1.08 \%$ & 28 & $\begin{array}{l}\text { linalool }(41.50 \%) \\
\text { linalyl anthranilate }(33.75 \%) \\
\alpha \text {-terpineol }(6.29 \%) \\
\text { geranyl acetate }(3.67 \%) \\
\text { nerol acetate }(2.09 \%) \\
\text { trans-geraniol }(2.07 \%)\end{array}$ & [126] \\
\hline $\mathrm{AP}$ & Dried & Tunisia & HD & GC-EIMS & $1.1 \%$ & 34 & $\begin{array}{l}\text { carvone }(40.8 \%) \\
\text { limonene }(20.8 \%) \\
1,8 \text {-cineole }(17.0 \%)\end{array}$ & [118] \\
\hline \multicolumn{9}{|c|}{ Mentha $\times$ rotundifolia $($ L.) Huds. } \\
\hline $\mathrm{AP}$ & Dried & Uruguay & HD & $\begin{array}{c}\text { GC } \\
\text { GC-MS }\end{array}$ & $1.02 \%$ & 23 & $\begin{array}{l}\text { piperitenone oxide }(80.8 \%) \\
\text { (Z)-sabinene hydrate }(2.0 \%) \\
\text { 4-terpineol }(1.5 \%)\end{array}$ & [112] \\
\hline \multicolumn{9}{|c|}{ Mentha $\times$ piperita $\mathrm{L}$. } \\
\hline $\mathrm{AP}$ & Fresh & Senegal & HD & $\begin{array}{c}\text { GC } \\
\text { GC-MS }\end{array}$ & & & $\begin{array}{l}\text { menthofuran }(30.7 \%) \\
\text { pulegone }(17.6 \%) \\
1.8 \text {-cineole }(3.7 \%) \\
\text { menthol }(15.9 \%) \\
\text { menthone }(13.0 \%)\end{array}$ & [119] \\
\hline $\mathrm{AP}$ & Dried & Senegal & HD & $\begin{array}{c}\text { GC } \\
\text { GC-MS }\end{array}$ & & & $\begin{array}{l}\text { menthofuran }(28.1 \%) \\
\text { pulegone }(13.8 \%) \\
1.8 \text {-cineole }(3.4 \%) \\
\text { menthol }(15.9 \%) \\
\text { menthone }(14.2 \%)\end{array}$ & [119] \\
\hline $\mathrm{AP}$ & Dried & Serbia & HD & GC-MS & ns & 26 & $\begin{array}{l}\text { menthol }(37.4 \%) \\
\text { menthyl acetate }(17.4 \%) \\
\text { menthone }(12.7 \%)\end{array}$ & [120] \\
\hline
\end{tabular}


Table 1. Cont.

\begin{tabular}{|c|c|c|c|c|c|c|c|c|}
\hline \multicolumn{3}{|c|}{ Plant } & \multicolumn{3}{|c|}{ Extraction } & \multicolumn{2}{|r|}{ Compounds } & \multirow{2}{*}{ References } \\
\hline Part & Material & Origin & Method & Identification & Yield & Number & Most Abundant & \\
\hline ns & ns & India & HD & $\begin{array}{l}\text { Oil: } \\
\text { GC and GC-MS } \\
\text { Oil vapours: } \\
\text { SPME } \\
\text { GC-MS }\end{array}$ & & $\begin{array}{c}\text { Oil: } 47 \\
\text { Vapour: } 18\end{array}$ & $\begin{array}{l}\text { Oil: } \\
\text {-menthol }(19.1 \%) \\
\text {-isomenthone }(14.8 \%) \\
\text {-limonene }(10.6 \%) \\
\text {-isomenthanol }(8.8 \%) \\
\text {-menthyl acetate }(6.6 \%) \\
\text { - } \beta \text {-pinene }(5.6 \%) \\
\text { Oil vapour: } \\
-\alpha \text {-pinene }(17.3 \%) \\
\text {-limonene }(18.4 \%) \\
\text { - } \beta \text {-pinene }(13.9 \%) \\
\text {-isomenthone }(9 \%) \\
\text {-menthyl acetate }(6.6 \%) \\
\text { - } \beta \text {-phellandrene }(5.8 \%)\end{array}$ & [127] \\
\hline $\mathrm{L}$ & Dried & Morocco & HD & $\begin{array}{c}\text { GC } \\
\text { GC-MS }\end{array}$ & $1.02 \%$ & 29 & $\begin{array}{l}\text { menthone }(29.01 \%) \\
\text { menthol }(5.58 \%) \\
\text { menthyl acetate }(3.34 \%) \\
\text { menthofuran }(3.01 \%) \\
1,8 \text {-cineole }(2.40 \%)\end{array}$ & [128] \\
\hline $\mathrm{L}$ & Dried & Iran & HD & $\begin{array}{c}\text { GC } \\
\text { GC-MS }\end{array}$ & $1.38 \%$ & 35 & $\begin{array}{l}\text { menthone }(30.63 \%) \\
\text { menthol }(25.16 \%) \\
\text { menthofuran }(6.47 \%) \\
\beta \text {-phellandrene }(5.59 \%) \\
\text { isomenthone }(4.74 \%) \\
\text { menthol acetate }(4.61 \%) \\
\text { pulegone }(4.39 \%) \\
\beta \text {-caryophyllene }(3.05 \%) \\
\text { neomenthol }(2.83 \%) \\
\text { 1,8-cineole }(2.15 \%)\end{array}$ & [129] \\
\hline $\mathrm{AP}$ & Dried & Iran & $\begin{array}{c}\text { HD } \\
\text { HS/SPME }\end{array}$ & $\begin{array}{c}\text { GC } \\
\text { GC-MS }\end{array}$ & $0.42 \%$ & $\begin{array}{c}\text { HD: } 39 \\
\text { HS/SPME: } \\
41\end{array}$ & $\begin{array}{l}\text { HD oil: } \\
\text {-menthol }(45.34 \%) \\
\text {-menthone }(16.04 \%) \\
\text {-menthofuran }(8.91 \%) \\
\text { HS/SPME oil: } \\
\text {-menthol }(29.38 \%) \\
\text {-menthone }(16.88 \%) \\
\text {-cis-carane }(14.39 \%) \\
\text {-menthofuran }(11.38 \%) \\
\text {-1,8-cineole }(9.45 \%) \\
\end{array}$ & [130] \\
\hline
\end{tabular}


Table 1. Cont.

\begin{tabular}{|c|c|c|c|c|c|c|c|c|}
\hline \multicolumn{3}{|c|}{ Plant } & \multicolumn{3}{|c|}{ Extraction } & \multicolumn{2}{|r|}{ Compounds } & \multirow{2}{*}{ References } \\
\hline Part & Material & Origin & Method & Identification & Yield & Number & Most Abundant & \\
\hline $\mathrm{L}$ & ns & China & HD & GC-MS & ns & 51 & $\begin{array}{l}\text { menthol }(30.69 \%) \\
\text { menthone }(14.51 \%) \\
\text { menthyl acetate }(12.86 \%) \\
\text { neomenthol }(9.26 \%) \\
\text { pulegone }(4.36 \%) \\
\text { cineole }(2.91 \%) \\
\text { caryophyllene }(2.52 \%)\end{array}$ & [131] \\
\hline $\mathrm{AP}$ & Fresh & Taiwan & $\mathrm{HD}$ & GC-MS & $0.3 \%$ & 10 & $\begin{array}{l}\text { menthol }(30.35 \%) \\
\text { menthone }(21.12 \%) \\
\text { trans-carane }(10.99 \%) \\
\text { isomenthol }(6.26 \%)\end{array}$ & [132] \\
\hline $\mathrm{AP}$ & Dried & $\begin{array}{l}\text { Saudi } \\
\text { Arabia }\end{array}$ & $\mathrm{HD}$ & GC-MS & ns & 19 & $\begin{array}{l}\text { menthol }(36.02 \%) \\
\text { menthone }(24.56 \%) \\
\text { menthyl acetate }(8.95 \%) \\
\text { menthofuran }(6.88 \%)\end{array}$ & [133] \\
\hline ns & ns & Brazil & SD & GC-MS & ns & 36 & $\begin{array}{l}\text { menthol }(59.73 \%) \\
\text { isomenthone }(18.45 \%) \\
\text { methyl acetate }(6.02 \%) \\
\text { neomenthol }(2.43 \%) \\
\text { isopulegol }(2.15 \%)\end{array}$ & [83] \\
\hline $\mathrm{L}$ & Dried & Oman & $\mathrm{HD}$ & GC-MS & ns & 14 & $\begin{array}{l}\text { carvone }(34.94 \%) \\
\text { pulegone }(14.89 \%) \\
\text { methyl petroselinate }(15.51 \%) \\
\text { D-limonene }(11.20 \%) \\
\text { p-cineole }(5.70 \%)\end{array}$ & [134] \\
\hline $\mathrm{AP}$ & Dried & Morocco & $\mathrm{SD}$ & $\begin{array}{c}\text { GC } \\
\text { GC-MS }\end{array}$ & $1.40 \%$ & 37 & $\begin{array}{l}\text { linalool (41.4\%) } \\
\text { linalyl acetate (39.5\%) }\end{array}$ & [115] \\
\hline $\mathrm{AP}$ & Dried & Brazil & HD & GC-MS & $0.10 \%$ & 18 & $\begin{array}{l}\text { D-carvone }(49.27 \%) \\
\text { limonene }(37.18 \%)\end{array}$ & [96] \\
\hline $\mathrm{AP}$ & Fresh & Senegal & HD & GC & $0.28 \%$ & 29 & $\begin{array}{l}\text { linalool }(45.8 \%) \\
\text { linalyl acetate }(42.7 \%) \\
1.8 \text {-cineole }(2.0 \%)\end{array}$ & [119] \\
\hline $\mathrm{AP}$ & Dried & Senegal & HD & GC-MS & $0.21 \%$ & 34 & $\begin{array}{l}\text { linalool }(42.0 \%) \\
\text { linalyl acetate }(38.5 \%) \\
\text { 1.8-cineole }(3.1 \%)\end{array}$ & [119] \\
\hline
\end{tabular}

AP, all plant; DHM, dichloromethane; EIMS, electron impact mass spectrometry; F, flowers; FID, flame ionization detector; GC, gas chromatography; HD, hydrodistillation; HS/SPME, headspace/solid-phase micro-extraction; IR, infrared spectroscopy; L, leaves; MS, mass spectrometry; NMR, nuclear magnetic resonance; ns, not specified; S, seeds; SD, steam distillation; SPME, solid phase micro extraction. 
On the other hand, $M$. arvensis essential oil was found to contain a lower number of chemical constituents (from 7 to 26) than the other members of Mentha genus. In the study carried out by Hussain et al. [135], menthol and isomenthone were the most abundant compounds.

Studies on M. canadensis essential oil, collected in China, Brazil and India and extracted using hydrodistillation, identified menthol as the main constituent $[96,98,99]$. Still, in the study performed in China, it was observed that salt stress can reduce menthol concentration at the same time increase menthone and pulegone contents in M. canadensis essential oil.

Regarding M. cervina essential oil, pulegone and isomenthone were the main constituents identified in all studies performed [85,101,102]. Rodrigues et al. [85] found that cultivated populations belonging to the same chemotype were characterized by the presence of pulegone as the most abundant constituent. Therefore, the studied essential oils contained in their chemical composition oxygenated monoterpenes, with pulegone being the main compound (52-75\%), followed by isomenthone, limonene and menthone. These findings allowed authors to classify the obtained oils into two categories: (1) pulegone-rich with low isomenthone content; and (2) pulegone- and isomenthone-rich oils.

Chowdhury et al. [136] investigated the essential oil chemical profile of different M. spicata varieties from Bangladesh, and found (-)-carvone and limonene as major constituents, with these data having been recently confirmed by Dwivedy et al. [100]. For Mentha diemenica essential oil, collected in Queensland (Australia), menthone, neomenthyl acetate and pulegone were found to be the main compounds present, while essential oil of the same species from Canada had significantly higher amounts of pulegone, clearly supporting it as the main compound, followed by menthone and isomenthone, with neomenthyl acetate not being found [103].

There are a number of studies dealing with the chemical composition of M. longifolia essential oils from different geographical origins, showing a great degree of chemical variation, which is not surprising, bearing in mind the very high morphological diversity of this species, resulting in a high number $(n=276)$ of subspecies, varieties and forms. The main constituents of M. longifolia essential oil belong to oxygenated monoterpenes group, which include pulegone [108], piperitenone oxide [107] and 1,8-cineole [105]. Beyond these, carvone, isomenthone, borneol, menthol, menthone, piperitenone, dihydrocarvone, limonene, sabinene, $\alpha$-pinene, eucalyptol, $\gamma$-terpineol, $\beta$-caryophyllene, isopulegone, cadinene and $\beta$-pinene were also recorded as meaningful compounds from $M$. longifolia essential oil.

Indeed, chemical profiling allows the differentiation of many chemotypes, each of them being dominated by one terpene, like piperotenone oxide, carvone, piperitone epoxide, menthone, pulegone, piperitone, trans-dihydrocarvone, isomenthone, menthofuran, menthol, 1,8-cineole, isopiperitenone, piperitenone and borneol [86,137-142].

For M. spicata subsp. condensata (Briq.) Greuter and Burdet there is only one study assessing their chemical composition, in which pulegone, piperitenone oxide and piperitenone were identified as the most abundant ones [123].

M. pulegium (pennyroyal) essential oil has been extensively studied [109-113,115]. Pulegone was the most abundant compound identified in all the studied essential oils. Other compounds present in relatively high amounts were menthone, piperitenone, piperitone, isomenthone and limonene. In a similar way, M. spicata (spearmint) essential oils also have several compounds present as main constituents, corresponding to up to $94.8 \%$ of the total oil content [87]. M. spicata chemotypes are characterized by (-)-carvone (40.8 to $67.8 \%)$ [96,108,113,115,116,118-120,122], piperitone oxide (35.7 to $94.8 \%$ ) [87,121], piperitone (81.2\%) [117] or pulegone (53.9\%) [96]. So far, series of M. suaveolens (grapefruit mint) essential oils chemotypes has been described, with predominance of pulegone [125], (-)-carvone [118,124], linalool [126], piperitone, piperitone oxide, menthone/isomenthone, pulegone/menthone/isomenthone, and pulegone/piperitone $[111,118,143]$. Oumzil et al. [125] analyzed M. suaveolens (grapefruit mint) essential oils collected in various regions of Morocco and concluded that three chemotypes can be defined: pulegone-rich, piperitenone oxide-rich or containing similar proportions of piperitenone and piperitone oxides. 
Mentha $\times$ rotundifolia (L.) Huds. is a species originating from M. longifolia and M. suaveolens hybridization. Twenty-three compounds were identified by Lorenzo et al. [112] in the essential oil of an Uruguayan population, where the main constituent (80.8\%) was piperitenone oxide.

Considering $M . \times$ piperita essential oil, studies showed a very high variability derived from the existence of numerous chemotypes. Barrosa et al. [96] studied two different $M$. $\times$ piperita varieties (chocolate mint and grapefruit mint varieties) and identified menthofuran, menthone $d$-neoisomenthol and pulegone as the main compounds in a chocolate mint variety, while linalyl acetate with linalool were those prevailing in a grapefruit mint variety. A commercial essential oil purchased in New Delhi (India) presented, in its chemical composition, menthol (19.1\%), isomenthone $(14.8 \%)$ and limonene $(10.6 \%)$ as major components [127]. M. $\times$ piperita essential oil collected in Serbia presented a similar composition to that collected in Morocco, with menthol, menthyl acetate and menthone as the main constituents $[120,128]$. Menthol was also found to be the main compound present in M. $\times$ piperita essential oils isolated from plant material collected in Iran, China, Taiwan, Saudi Arabia and Brazil [129-133]. However, beyond menthol (up to 59.7\%), high amounts of menthone, isomenthone, menthyl acetate and menthofuran were also reported. Another chemotype identified from this species is characterized by its high amount of (-)-carvone, accompanied by limonene as the second main constituent [134].

\subsection{Non-Volatile Compounds}

A wide range of other chemical constituents, mostly phenolic compounds, are also present in mint tissues [144], as briefly shown in Table 2. Interestingly, it should be highlighted that rosmarinic acid, luteolin-7-O-glucoside, salvianolic acid, eriocitrin and hesperidin have been found to be the major non-volatile constituents in Mentha species [145].

Table 2. Major non-volatile compounds in Mentha species.

\begin{tabular}{ll}
\hline Chemical Constituents & \multicolumn{1}{c}{ Individual Compounds } \\
\hline Anthocyanidins & Cyanidin, delphinidin, luteolinidin, pelargonidin, petunidin \\
\hline Coumarins & Esculetin and scopoletin \\
\hline Flavanols & Catechin, epicatechin \\
\hline Flavanones & Eriocitrin, eriodictyol, hesperidin, naringenin, narirutin \\
\hline Flavones & $\begin{array}{l}\text { Apigenin, diosmetin, diosmin, luteolin, luteolin-O-glucuronide, gardenin B, } \\
\text { luteolin-O-glucoside, pebrellin, salvigenin, thymusin, thymonin }\end{array}$ \\
\hline $\begin{array}{l}\text { Flavonols and } \\
\text { dihydroflavonols }\end{array}$ & Quercetin, kaempferol, rutin \\
\hline Phenolic acids & $\begin{array}{l}\text { Cinnamic acid, its analogs (hydroxybenzoic, } p \text {-coumaric, ferulic, caffeic, sinapic, rosmarinic, } \\
\text { salvianolic, isosalvianolic, didehydrosalvianolic and lithospermic acids, nepetoidin A/B) and } \\
\text { glycosides (caffeic acid glucuronide, chlorogenic, caftaric), gallic, syringic and vanillic acids }\end{array}$ \\
\hline Phenylethanoids & Tyrosol \\
\hline Stilbenoids & Resveratrol \\
\hline Terpenes & Oleanolic acid \\
\hline
\end{tabular}

\section{Food Preservative Applications of Genus Mentha Essential Oils}

Food manufacturers, regulatory agencies and, finally, consumers have been increasingly concerned about microbiological food safety and the growing number of foodborne illnesses caused by pathogens [146]. According to the World Health Organization's (WHO) global estimates of foodborne diseases, 600 million people fall ill after consuming contaminated food, of which 420,000 die. In European countries alone, more than 23 million people fall ill every year from unsafe food, and this results in 5,000 deaths [147]. Over 90\% of all cases of food poisoning are caused by Bacillus cereus, Campylobacter jejuni, Clostridium botulinum, Clostridium perfringens, Escherichia coli pathogenic strains, Listeria monocytogenes, Salmonella spp., Shigella spp., Staphylococcus aureus, Yersinia enterocolitica, as well 
as Vibrio cholerae [148]. These microorganisms are responsible for foodborne outbreaks in different food branches, such as drinking water, beverages, dairy products, fruits, vegetables and even meat and fish products [149]. For decades, in the food industry, a wide variety of synthetic compounds has been used for preservative and antimicrobial purposes with the aim of inhibiting microorganism growth and spoiling. Sodium benzoate, sodium and calcium propionate, sorbic acid, ethyl formate, and sulfur dioxide are examples of chemical substances commercially used to inhibit the growth of microorganisms in foods [150]. Butylated hydroxyanisole (BHA), butylated hydroxytoluene (BHT), tertiary butylated hydroquinone (TBHQ), propyl gallate, ascorbic acid (vitamin C) and tocopherols (vitamin E) are antioxidants used as food preservatives [150]. However, it has also been stated that the widespread use of synthetic preservatives has led to huge ecological and medical problems, which, in addition to economic considerations, have triggered the search for new/safer strategies against microbiota spoilage [151,152]. Due to the growing consumer demand for safe, high-quality and healthy food with reduced quantity of synthetic preservatives or antimicrobials, an increasing interest has been stated on natural antimicrobials from plants [153]. It has long been known that plant phytochemicals protect against viruses, bacteria, fungi and herbivores, but it has only recently been learned that they can be also used for food spoilage microorganism protection.

One of the most popular and representative plant groups is the Lamiaceae family. Nowadays, it is used both in traditional and modern medicine, as well as in the pharmaceutical and food industries. The use of mint is not strictly limited to essential oils, which are widely recognized for their strong aromatic properties. Indeed, essential oils and their derived extracts can be effectively used as natural food preservatives. As a result, they can fulfill several important tasks: prolong shelf-life, eliminate synthetic preservatives and food flavors, as well as forming part of a healthy food trend that influences market sales. Due to the overall popularity and occurrence, mint appear to be relatively well tested in terms of antibacterial activity against a wide spectrum of bacteria, such as E. coli pathogenic strains, $L$. monocytogenes, Salmonella spp., S. aureus, and many others (Table 3). Curiously, the vast majority of studies on Mentha spp. antimicrobial effects have been linked to essential oils and plant extracts.

\subsection{In vitro Studies on Mentha Genus}

\subsubsection{Extracts}

Methanolic extracts from six Mentha species (M. aquatica, M. arvensis, M. × piperita, M. pulegium, M. $\times$ rotundifolia and $M . \times$ villosa Huds.) exhibited a powerful antioxidant activity, ranging from 7.5 to $44.7 \mu \mathrm{g} / \mathrm{mL}$, which supports their upcoming use as natural food preservatives [154]. For example, M. $\times$ piperita extract, rich in flavonoids, at a concentration of $5 \mathrm{mg} / \mathrm{mL}$ reduced the in vitro growth of two cereal fungi, Phoma sorghina and Fusarium moniliforme around $72 \%$ and $55 \%$, respectively; dimethylsulfoxide (DMSO) at $1 \%$ DMSO was added to the culture media as a control [155]. Sujana et al. [156] reported that M. $\times$ piperita leaf extract is more active against S. aureus, Bacilus subtilis and Proteus vulgaris than E. coli (positive control: chloramphenicol $100 \mu \mathrm{g} / \mathrm{mL}$ ). Bayoub et al. [157] reported that the minimal inhibitory concentration (MIC) of $M$. suaveolens ethanol extract against Listeria monocytogenes subsp. Timija was significantly lower $(0.3 \mathrm{mg} / \mathrm{mL})$ than MIC obtained for cinnamon $(0.4 \mathrm{mg} / \mathrm{mL})$, cistus $(0.5 \mathrm{mg} / \mathrm{mL})$, rose $(0.9 \mathrm{mg} / \mathrm{mL})$, thyme $(1.6 \mathrm{mg} / \mathrm{mL})$, wild thyme $(2.2 \mathrm{mg} / \mathrm{mL})$, artemisia $(3.8 \mathrm{mg} / \mathrm{mL})$, rosemary $(5.3 \mathrm{mg} / \mathrm{mL})$, geranium $(6.2 \mathrm{mg} / \mathrm{mL})$, chamomile $(6.8 \mathrm{mg} / \mathrm{mL})$, lavender $(11.5 \mathrm{mg} / \mathrm{mL})$ and verbena $(11.8 \mathrm{mg} / \mathrm{mL})$ (positive controls: penicillin $\mathrm{G}$ 10 units, nalidixic acid $30 \mu \mathrm{g}$, vancomycin $30 \mu \mathrm{g}$, tetracycline $30 \mu \mathrm{g}$, chloramphenicol $30 \mu \mathrm{g}$, novobiocin $5 \mu \mathrm{g}$, and ampicillin 5 and $30 \mu \mathrm{g}$ ). Moreover, Bupesh et al. [158] found prominent antipathogenic effects of peppermint leaf water extract against B. subtilis, Pseudomonas aeruginosa, Staphylococcus aureus and Serratia marcescens by an in vitro agar well diffusion method (positive control: chloramphenicol $100 \mu \mathrm{g} / \mathrm{mL}$ ). Dhiman et al. [159] tested the effect of different extracts from M. arvensis prepared using different solvents (acetone, methanol, ethanol, water) against microorganisms isolated from spoiled juices: B. cereus, Serratia spp., Rhodotorula mucilaginosa, Aspergillus flavus and Penicillium citrinum. 
The obtained results showed that ethanol extract activity was similar to that of sodium benzoate $(100 \mathrm{mg} / \mathrm{mL})$, a commonly used preservative in beverage production. Beyond direct antimicrobial activity, M. $\times$ piperita essential oils and extracts also inhibited L. monocytogenes, P. aeruginosa, Asaia lannensis, Asaia bogorensis and Candida species (C. albicans and C. dubliniensis) biofilm formation and development $[160,161]$. The final concentration of the extracts in the wells was $1 \mathrm{mg} / \mathrm{mL}$, while ciprofloxacin at a concentration of $2,5 \mu \mathrm{g} / \mathrm{mL}$ and amphotericin $\mathrm{B}$ at $5 \mu \mathrm{g} / \mathrm{mL}$ were used as the positive controls for $P$. aeruginosa and C. albicans, respectively [160]; for the other microorganisms, the control samples were the same culture media but without $M . \times$ piperita [161]. Panda et al. [162] showed that $M$. arvensis aqueous extract at $0.8 \mathrm{mg} / \mathrm{mL}$ inhibited citrinin production by P. citrinum up to $73 \%$ (positive control: none).

Table 3. Mentha spp. activity against bacterial pathogens tested in vitro.

\begin{tabular}{|c|c|c|}
\hline Plant Species & Bacterial Strain & References \\
\hline \multirow{16}{*}{ Mentha $\times$ piperita $\mathbf{L}$. } & Gram negative: & \multirow{16}{*}[156,158,163-169]{} \\
\hline & Proteobacteria & \\
\hline & Escherichia coli & \\
\hline & Klebsiella pneumoniae & \\
\hline & Proteus mirabilis, $P$. vulgaris & \\
\hline & Pseudomonas aeruginosa & \\
\hline & Salmonella enteritidis, S. paratyphi A and B, S. pullorum, & \\
\hline & S. typhi, S. typhimurium & \\
\hline & Shigella dysenteriae & \\
\hline & Yersinia enterocolitica & \\
\hline & Gram positive: & \\
\hline & Firmicutes & \\
\hline & Bacillus cereus, B. subtilis & \\
\hline & Listeria monocytogenes & \\
\hline & Staphylococcus aureus & \\
\hline & Streptococcus pyogenes & \\
\hline \multirow{10}{*}{ Mentha suaveolens $\mathrm{L}$. } & Gram negative: & \multirow{10}{*}{ [125] } \\
\hline & Proteobacteria & \\
\hline & Escherichia coli & \\
\hline & Klebsiella pneumoniae & \\
\hline & Pseudomonas aeruginosa & \\
\hline & Proteus mirabilis & \\
\hline & Gram positive: & \\
\hline & Firmicutes & \\
\hline & Bacillus anthracis & \\
\hline & Staphylococcus aureus & \\
\hline \multirow{13}{*}{ Mentha spicata $\mathbf{L}$. } & Gram negative: & \multirow{13}{*}[118,170,171]{} \\
\hline & Proteobacteria & \\
\hline & Escherichia coli & \\
\hline & Klebsiella pneumoniae & \\
\hline & Proteus mirabilis & \\
\hline & Pseudomonas aeruginosa & \\
\hline & Salmonella typhimurium & \\
\hline & Vibrio spp. & \\
\hline & Gram positive: & \\
\hline & Firmicutes & \\
\hline & Bacillus cereus, B. subtilis & \\
\hline & Listeria monocytogenes & \\
\hline & Staphylococcus aureus & \\
\hline \multirow{8}{*}{$\begin{array}{l}\text { Mentha } \times \text { rotundifolia } \\
\text { (L.) Huds. }\end{array}$} & Gram negative: & \multirow{8}{*}{ [172] } \\
\hline & Proteobacteria & \\
\hline & Escherichia coli & \\
\hline & Salmonella typhimurium & \\
\hline & Gram positive: & \\
\hline & Firmicutes & \\
\hline & Bacillus cereus & \\
\hline & Staphylococcus aureus & \\
\hline
\end{tabular}


Table 3. Cont.

\begin{tabular}{|c|c|c|}
\hline Plant Species & Bacterial Strain & References \\
\hline \multirow{9}{*}{ Mentha arvensis L. } & Gram negative: & \multirow{9}{*}{ [173] } \\
\hline & Proteobacteria & \\
\hline & Escherichia coli & \\
\hline & Klebsiella pneumoniae & \\
\hline & Pseudomonas aeruginosa & \\
\hline & Shigella flexneri & \\
\hline & Gram positive: & \\
\hline & Firmicutes & \\
\hline & Staphylococcus aureus & \\
\hline \multirow{11}{*}{ Mentha longifolia $\mathrm{L}$. } & Gram negative: & \multirow{11}{*}[171,174]{} \\
\hline & Proteobacteria & \\
\hline & Escherichia coli & \\
\hline & Pseudomonas aeruginosa & \\
\hline & Salmonella typhimurium & \\
\hline & Gram positive: & \\
\hline & Firmicutes & \\
\hline & Bacillus cereus & \\
\hline & Listeria monocytogenes & \\
\hline & Staphylococcus aureus & \\
\hline & Streptococcus pyogenes & \\
\hline \multirow{9}{*}{ Mentha pulegium $\mathrm{L}$. } & Gram negative: & \multirow{9}{*}[175,176]{} \\
\hline & Proteobacteria & \\
\hline & Escherichia coli & \\
\hline & Pseudomonas aeruginosa & \\
\hline & Salmonella typhimurium & \\
\hline & Gram positive: & \\
\hline & Firmicutes & \\
\hline & Bacillus cereus & \\
\hline & Staphylococcus aureus & \\
\hline
\end{tabular}

\subsubsection{Essential Oils}

Essential oils derived from different mint species have shown significant antibacterial activity against human pathogenic microorganisms, such as S. aureus, Micrococcus flavus, B. subtilis, Staphylococcus epidermidis and Salmonella enteritidis [133]. M. $\times$ piperita essential oil, with (-)-carvone $(35 \%)$, pulegone $(15 \%)$, methyl petroselinate $(16 \%)$ as main compounds, demonstrated antimicrobial activity against Gram-positive (S. aureus) and Gram-negative (E. coli) bacteria [134]. Essential oils from $M$. aquatica, $M$. longifolia or $M . \times$ piperita aerial parts were markedly active against pathogenic fungi, like C. albicans, Epidermophyton floccosum, Microsporum canis and Trichophyton spp. (T. mentagrophytes, T. rubrum and T. tonsurans) $[138,177] . \quad$ M. $\times$ piperita essential oil showed significant antifungal activity against $A$. alternata, Fusarium tabacinum, Penicillum spp., Fusarium oxyporum and Aspergillus fumigatus [133]. Studies conducted by Mahboubi and Haghi [178] reported that M. pulegium essential oil exhibited antimicrobial activity against $S$. aureus, L. monocytogenes, B. cereus, E. coli and yeast $C$. albicans. The data obtained by the authors pointed out that $M$. pulegium essential oil activity was comparable to well-known antibiotics vancomycin, erythromycin or gentamycin. In a similar way, Ait-Ouazzou et al. [179] highlighted that M. pulegium essential oil antibacterial activity against S. aureus, L. monocytogenes, S. enteritidis and E. coli was higher than galingale (Cyperus longus) and juniper (Juniperus phoenicea). Dhifi et al. [180] also reported that $M$. spicata essential oil showed high activity against Gram-positive Staphylococcus (S. epidermidis and S. aureus) and Gram-negative Salmonella and E. coli species. Soković et al. [181] found that M. $\times$ piperita and M. spicata essential oils were more active against pathogenic bacteria B. subtilis, E. coli, Pseudomonas (P. mirabilis, P. aeruginosa), Salmonella (S. enteritidis, S. typhimurium) and S. aureus spp. than essential oils from sweet basil (Ocimum basilicum L.), lavender (Lavandula angustifolia Mill.), bitter orange (Citrus $\times$ aurantium L.), sage (Salvia officinalis 
L.) and chamomile (Matricaria chamomilla L.). The authors also noted that menthol was more active than linalyl acetate, limonene, $\beta$-pinene, $\alpha$-pinene, camphor, linalool and 1,8-cineole.

On the other hand, Ben Arfa et al. [182] compared the antimicrobial activity of carvacrol with that of carvacrol methyl ether, carvacrol acetate, eugenol, and menthol. Menthol showed very weak antibacterial activity, with it being suggested that the benzene ring is of huge importance to promoting strong effects. Indeed, menthol molecules have a cyclohexane ring, which seems to make this compound less active. Nonetheless, compounds present in essential oils and extracts show multiple actions and even synergistic effects. Consequently, mixtures can be more effective as food preservatives than pure substances. The main mechanisms of antimicrobial synergy include: (1) sequential steps inhibition in specific biochemical pathways; (2) inhibition of enzymes that degrade of excrete antimicrobial agents; and (3) cell wall/membrane interaction leading to increased uptake of other antimicrobials [183]. For example, in yeasts, the results obtained by Ferreira et al. [184] indicated that M. × piperita essential oil was able to induce cell death in Saccharomyces cerevisiae due to pro-oxidant effects, both in cytosol and mitochondria.

Synergy can not only be observed between different constituents of one essential oil or extract but also in a mixture of them. For example, the combination of Lippia multiflora Moldenke and M. $\times$ piperita essential oils showed synergetic effects against E. coli, Enterococcus faecalis, Enterobacter aerogenes, L. monocytogenes, P. aeruginosa, Shigella dysenteriae, S. aureus and Salmonella (S. enterica and S. typhimurium) species [185]. Soković et al. [181] and Riahi et al. [172] indicated that, in general, essential oils show stronger action against Gram-positive than Gram-negative bacteria. In fact, it has been shown that the lower susceptibility of Gram-negative bacteria results from the presence of hydrophobic lipopolysaccharide in their outer cell membrane, which confers protection against different active agents [170]. This bacterial structure prevents depolarization and pore formation, while at the same time increasing membrane permeability or fluidifying effect [183].

There are some crucial factors affecting antimicrobial activity, including essential oil composition, active substances concentration and type of microorganisms tested. First of all, essential oil composition may be determined by some plant cultivation factors, such as environmental conditions, harvesting period, drying method, storage conditions as well as extraction methods [186]. Although the different mint varieties from which plant extracts and essential oils are derived are characterized by strong and multifaceted in vitro activities against bacteria, yeasts and molds, it should be clearly emphasized that their biological activity may vary considerably in industrial conditions for specific food matrices.

Table 4 lists in vitro Mentha species activities against fungi and yeasts. Results from in vitro studies for M. × piperita essential oils against several Aspergillus (A. flavus, A. fumigatus, A. oryzae, A. clavatus), Candida (C. albicans, C. glabrata, C. tropicalis, C. krusei, C. dubliniensis, C. parapsilosis) and Cryptococcus neoformans species showed prominent effects at relatively low concentrations, ranging from 0.5 to $4 \mu \mathrm{L} / \mathrm{mL}$ [187]. According Pandey et al. [188], M. arvensis essential oil displayed a high toxicity on Penicillium italicum than lemon grass (Cymbopogon citratus) and sweet basil (O. basilicum) essential oils. Mentha spp. essential oils used for Aspergillus parasiticus control inhibited aflatoxin synthesis [189]. In the work performed by Kumar et al. [190], M. arvensis essential oil was tested against 9 postharvest fungi and exhibited absolute inhibition of Aspergillus species (A. flavus, A. fumigatus, A. ochraceus, A. niger, A. terreus), Helminthosporium oryzae and Sclerotium rolfsii. Still, essential oil significantly exhibited growth in all tested fungi at a concentration of $500 \mathrm{ppm}$, with the observed activity being similar to basil (Ocimum americanum L.) essential oil [191]. Soković et al. [120] investigated M. spicata, M. $\times$ piperita and thymes (Thymus vulgaris L. and T. sibthorpii Benth.) activity against 17 plant, animal and human pathogens. Results showed that $M$. spicata essential oil had a greater fungistatic activity than M. $\times$ piperita, but a weaker one than T. vulgaris. In addition, the authors found that all tested essential oils showed stronger antimicrobial activities than bifonazole against $A$. alternata, Aspergillus (A. niger, A. ochraceus, A. versicolor, A. flavus, A. terreus), Cladosporium cladosporioides, Fusarium tricinctum, Penicillium (P. ochrochloron, P. funiculosum), Phomopsis helianthi, Trichoderma viride, and Trichophyton (T. mentagrophytes, T. rubrum, T. tonsurans) species. 
Table 4. Mentha spp. activity against fungi and yeasts tested in vitro.

\begin{tabular}{|c|c|c|}
\hline Plant Species & Yeast/Fungi Strain & References \\
\hline Mentha arvensis $\mathrm{L}$. & Penicillium citrinum & [162] \\
\hline Mentha longifolia L. & Candida albicans & [174] \\
\hline \multirow[b]{2}{*}{ Mentha $\times$ piperita $\mathbf{L}$. } & Candida albicans & [172] \\
\hline & $\begin{array}{c}\text { Aspergillus flavus } \\
\text { Aspergillus } \\
\text { parasiticus } \\
\text { Fusarium solani } \\
\text { Sclerotium rolfsii } \\
\text { Candida albicans }\end{array}$ & [168] \\
\hline Mentha $\times$ rotundifolia (L.) Huds. & Candida albicans & [172] \\
\hline Mentha suaveolens L. & Candida albicans & [125] \\
\hline $\begin{array}{c}\text { Mentha } \times \text { piperita essential oils in chitosan-cinnamic acid } \\
\text { nanogel }\end{array}$ & Aspergillus flavus & [192] \\
\hline
\end{tabular}

\section{Shelf-Life Prolongation}

Despite Mentha spp. plant extracts and essential oils having shown great in vitro antimicrobial effects against bacteria, yeasts and molds, their action can be quite different in complex environments, such as food matrices. Indeed, essential oils' biological activity is markedly influenced by food components (e.g., fats, carbohydrates, proteins, water, salt, preservatives), temperature, $\mathrm{pH}$, water activity and packaging methods [193]. The negative impact of high protein content on essential oil activity was reported by Tassou et al. [163], who studied the effect of mint essential oils on S. enteritidis and $S$. aureus growth. The authors attributed antimicrobial activity reduction to protein content, suggesting that the phenolic group monoterpenes could bind to proteins, thereby lowering the number of antimicrobial compounds available. Cava et al. [194] tested the antimicrobial activity of mint essential oils against L. monocytogenes in milk, and found that essential oils bioactivity was reduced by fats. Diverse studies reported that essential oils exhibited highest activity at low pH levels [195,196]. However, other parameters also affect essential oil activity, like temperature and sodium chloride. Combination of carvacrol and $p$-cymene with sodium chloride $(1.3 \mathrm{~g} / \mathrm{L})$ showed an antagonistic effect. On the other hand, it is well known that high sodium chloride concentration contributes to cell lysis. Going further, essential oils show better antimicrobial activity in vapor than in liquid phase [197].

Both extracts and essential oils derived from different mint species may extend food products shelf-life (Table 5).

M. longifolia leaf extract (6.0\%) application shows better antioxidant and antimicrobial activities than cumin (Cuminum cyminum L.) seed extract. Wild mint extracts significantly affected fresh rainbow trout shelf-life, extending it by up to 12-18 days during storage in a refrigerator, which suggests that they can be effectively applied as natural preservatives in fish products shelf-life extension. Sensory analysis showed that rainbow trout treatment with $M$. longifolia extract improved overall quality and sensory properties [204].

Results obtained by Tassou et al. [210] showed that $M . \times$ piperita essential oil at concentrations ranging from 0.5 to $2.0 \%$ completely reduced S. enteritidis number in tzatziki, and markedly decreased its number in taramosalata. In the same study, L. monocytogenes populations showed a declining trend in a one-week storage period. However, this effect was not observed for pâté. The authors speculated that mint essential oils' antibacterial action depends not only on essential oil concentration, but also on food product (type, $\mathrm{pH}$, storage temperature) and type of spoilage microbiota. Klūga et al. [198], reported that $M . \times$ piperita leaf extract protected fish from spoilage. Also, mint extract treatment suppressed total Enterobacteriaceae and Pseudomonas spp. bacteria growth in mackerel. Moreover, in these food matrices, mint extract inhibited lipid oxidation, and then enhanced stability storage and extended shelf 
life by 2 to 5 days [201]. Nonetheless, mint essential oils and extracts can also be successfully used in other food products. The study of Choi et al. [199] demonstrated peppermint oil antibacterial activities against Acidovorax citrulli, a bacteria responsible for watermelon blotch. These results suggested the possibility of using peppermint oil as an antibacterial agent to treat contaminated seeds. In addition to that, a mint-supplemented cereal biscuit (in different forms) enriched in natural antioxidants, maintained acceptable for consumption over 5 months storage period. Polyphenols' antioxidant efficiency prevented biscuit rancidity onset during storage; therefore, mint may be conceived of as a key substitute for synthetic antioxidants in baked food product preservation [203].

Table 5. Mentha spp. essential oil or extract application and food shelf-life prolongation.

\begin{tabular}{|c|c|c|c|}
\hline Plant Species & Spoiling Microorganisms & Food Matrix & Reference \\
\hline Mentha $\times$ piperita $\mathrm{L}$. & $\begin{array}{c}\frac{\text { Bacteria }}{\text { Gram negative: }} \\
\text { Proteobacteria } \\
\text { Acinetobacter pittii } \\
\text { Acinetobacter baumannii } \\
\text { Buttiauxella agrestis } \\
\text { Delftia acidovorans } \\
\text { Enterobacter cloacae } \\
\text { Escherichia coli } \\
\text { Lelliottia amnigena } \\
\text { Pantoea agglomerans } \\
\text { Pseudomonas alcaligenes } \\
\text { Pseudomonas oryzihabitans } \\
\text { Providencia rettgeri } \\
\text { Rahnella aquatilis } \\
\text { Serratia liquefaciens } \\
\text { Gram positive: }\end{array}$ & Fish & [198] \\
\hline Mentha $\times$ piperita $\mathrm{L}$. & $\begin{array}{l}\frac{\text { Bacteria }}{\text { Gram negative: }} \\
\text { Proteobacteria } \\
\text { Acidovorax citrulli } \\
\text { (bacterial fruit blotch) }\end{array}$ & Watermelon & [199] \\
\hline Mentha $\times$ piperita $\mathrm{L}$. & $\begin{array}{l}\frac{\text { Bacteria }}{\text { Gram negative: }} \\
\text { Proteobacteria } \\
\text { Escherichia coli }\end{array}$ & $\begin{array}{l}\text { Commercial } \\
\text { chicken soup }\end{array}$ & [200] \\
\hline $\begin{array}{l}\text { Mentha arvensis L. with } \\
\text { citrus peel extract }\end{array}$ & Aerobic plate count & Mackerel & [201] \\
\hline $\begin{array}{l}\text { Mentha spicata } \mathrm{L} . \\
\text { Mentha pulegium } \mathrm{L} \text {. }\end{array}$ & $\frac{\text { Fungi }}{\text { Debaryomyces hansenii }}$ & Doogh & [202] \\
\hline Mentha spicata $\mathrm{L}$. & Antioxidant properties & Biscuits & [203] \\
\hline Mentha longifolia $\mathrm{L}$. & $\begin{array}{l}\text { viable aerobic bacteria, } \\
\text { psychrotrophic bacteria }\end{array}$ & $\begin{array}{l}\text { Rainbow } \\
\text { trout (fish) }\end{array}$ & [204] \\
\hline Mentha pulegium $\mathrm{L}$. & $\begin{array}{l}\frac{\text { Bacteria }}{\text { Gram positive: }} \\
\text { Firmicutes } \\
\text { Listeria monocytogenes }\end{array}$ & White cheese & [205] \\
\hline
\end{tabular}


Table 5. Cont.

\begin{tabular}{|c|c|c|c|}
\hline Plant Species & Spoiling Microorganisms & Food Matrix & Reference \\
\hline $\begin{array}{l}\text { Mint essential oil } \\
\text { combined with HPP } \\
\text { process }\end{array}$ & $\begin{array}{l}\frac{\text { Bacteria }}{\text { Gram positive: }} \\
\text { Firmicutes } \\
\text { Listeria monocytogenes } \\
\text { Listeria innocua }\end{array}$ & $\begin{array}{l}\text { Yogurt drink } \\
\text { (ayran) }\end{array}$ & [206] \\
\hline \multirow[t]{2}{*}{ Mint extract } & Total count & Tomato juice & [207] \\
\hline & $\begin{array}{l}\frac{\text { Bacteria }}{\text { Gram negative: }} \\
\text { Proteobacteria } \\
\quad \text { Escherichia coli } \\
\quad \text { Staphylococcus typhimurium }\end{array}$ & $\begin{array}{l}\text { Fresh-cut } \\
\text { lettuce and } \\
\text { purslane }\end{array}$ & [208] \\
\hline Mint powder & $\begin{array}{l}\text { Standard plate count } \\
\text { Yeast/mould count }\end{array}$ & Chicken slices & [209] \\
\hline
\end{tabular}

Finally, essential oil synergy is also an interesting point [31]. M. $\times$ piperita essential oil and silver ions $\left(\mathrm{Ag}^{+}\right)$combination acted synergistically against $E$. coli, S. aureus and C. albicans cultures [211]. Also, M. $\times$ piperita essential oil $(0.5 \%)$ and bacteriocin $(1000 \mathrm{AU} / \mathrm{g})$ combination delayed microorganism spoilage proliferation in stored minced beef meat. Thus, biopreservative effect in combination can be considered a promising tool for upcoming application in meat products preservation [212].

\section{Conclusions and Future Perspectives}

Among Lamiaceae family, the Mentha genus encompasses several species already used at industrial scale and with well-developed cultivation. Extracts are traditionally used as food, and contain remarkable antioxidant phenolic compounds. Many essential oil chemotypes show distinct aromatic flavor conferred by different terpenes proportions. Both extracts and essential oils show activities on a broad spectrum of microorganisms tested in vitro as well as using various food matrices. Due to its natural origin, antioxidant and antimicrobial activities, mint-derived products could become a great alternative to artificial preservatives, and to find a wide range of applications for shelf-life extension of fruits and vegetables, beverages, dairy products, baking or meat and fish products. Nevertheless, industrial implementation depends on efficacy, ease of use and profitability of such natural additive over synthetic preservatives.

Extracts could be obtained as essential oil by-products, allowing a higher profitability, and where its moderate activity can be compensated by their safety. Besides that, essential oil taste is not neutral, and therefore its wide aroma should be carefully adapted to the matrices used in order to minimize the aromatic impact, and might also be used for food ingredient preservation before processing. Not least important to emphasize is that the finished products could also be marketed with the explicit mention of such natural preservatives to facilitate overall acceptance and demand. Further organoleptic studies should be performed, along with antimicrobial studies to assess the relevance of such ingredient in finished food products.

Author Contributions: All authors (B.S., Z.S.-R., J.M., F.S., H.A., D.K., S.S., M.S.-R., K.A., R.S.-R., M.M., A.S., N.M., and J.S.-R.) contributed equally to this work. M.S.-R., M.M. N.M., and J.S.-R. critically reviewed the manuscript. All the authors read and approved the final manuscript.

Funding: This work was supported by the Vice-chancellor for Research Affairs of Shahid Beheshti University of Medical Sciences, Tehran, Iran and Vicerrectoría de Investigación y Desarrollo from University of Concepción, Chile (216.073.031-1.0IN and 217.073.033-1.0). A. Sureda was supported by the Programme of Promotion of Biomedical Research and Health Sciences (CIBEROBN CB12/03/30038). 
Acknowledgments: N.M. thank to Portuguese Foundation for Science and Technology (FCT-Portugal) for the Strategic project ref. UID/BIM/04293/2013 and "NORTE2020-Programa Operacional Regional do Norte" (NORTE-01-0145-FEDER-000012).

Conflicts of Interest: The authors declare no conflict of interest.

\section{References}

1. Sharifi-Rad, J.; Tayeboon, G.S.; Niknam, F.; Sharifi-Rad, M.; Mohajeri, M.; Salehi, B.; Iriti, M.; Sharifi-Rad, M. Veronica persica poir. Extract-antibacterial, antifungal and scolicidal activities, and inhibitory potential on acetylcholinesterase, tyrosinase, lipoxygenase and xanthine oxidase. Cell. Mol. Biol. (Noisy-le-Grand France) 2018, 64, 50-56. [CrossRef]

2. Sharifi-Rad, J.; Iriti, M.; Setzer, W.N.; Sharifi-Rad, M.; Roointan, A.; Salehi, B. Antiviral activity of Veronica persica Poir. on herpes virus infection. Cell. Mol. Biol. (Noisy-le-Grand France) 2018, 64, 11-17. [CrossRef]

3. Sharifi-Rad, J.; Ayatollahi, S.A.; Varoni, E.M.; Salehi, B.; Kobarfard, F.; Sharifi-Rad, M.; Iriti, M.; Sharifi-Rad, M. Chemical composition and functional properties of essential oils from Nepeta schiraziana Boiss. Farmacia 2017, $65,802-812$.

4. Sharifi-Rad, M.; Nazaruk, J.; Polito, L.; Morais-Braga, M.F.B.; Rocha, J.E.; Coutinho, H.D.M.; Salehi, B.; Tabanelli, G.; Montanari, C.; del Mar Contreras, M. Matricaria genus as a source of antimicrobial agents: From farm to pharmacy and food applications. Microbiol. Res. 2018, 215, 76-88. [CrossRef] [PubMed]

5. Mishra, A.P.; Saklani, S.; Salehi, B.; Parcha, V.; Sharifi-Rad, M.; Milella, L.; Iriti, M.; Sharifi-Rad, J.; Srivastava, M. Satyrium nepalense, a high altitude medicinal orchid of indian himalayan region: Chemical profile and biological activities of tuber extracts. Cell. Mol. Biol. (Noisy-le-Grand France) 2018, 64, $35-43$. [CrossRef]

6. Salehi, B.; Sharopov, F.; Martorell, M.; Rajkovic, J.; Ademiluyi, A.; Sharifi-Rad, M.; Fokou, P.; Martins, N.; Iriti, M.; Sharifi-Rad, J. Phytochemicals in Helicobacter pylori infections: What are we doing now? Int. J. Mol. Sci. 2018, 19, 2361. [CrossRef] [PubMed]

7. Atanasov, A.G.; Waltenberger, B.; Pferschy-Wenzig, E.-M.; Linder, T.; Wawrosch, C.; Uhrin, P.; Temml, V.; Wang, L.; Schwaiger, S.; Heiss, E.H.; et al. Discovery and resupply of pharmacologically active plant-derived natural products: A review. Biotechnol. Adv. 2015, 33, 1582-1614. [CrossRef] [PubMed]

8. Yoo, S.; Kim, K.; Nam, H.; Lee, D. Discovering health benefits of phytochemicals with integrated analysis of the molecular network, chemical properties and ethnopharmacological evidence. Nutrients 2018, 10, 1042. [CrossRef] [PubMed]

9. Mosquera, C.; Panay, A.; Montoya, G. Pentacyclic triterpenes from Cecropia telenitida can function as inhibitors of 11 $\beta$-hydroxysteroid dehydrogenase type 1. Molecules 2018, 23, 1444. [CrossRef] [PubMed]

10. Malterud, K. Ethnopharmacology, chemistry and biological properties of four Malian medicinal plants. Plants 2017, 6, 11. [CrossRef] [PubMed]

11. Sharifi-Rad, M.; Fokou, P.V.T.; Sharopov, F.; Martorell, M.; Ademiluyi, A.O.; Rajkovic, J.; Salehi, B.; Martins, N.; Iriti, M.; Sharifi-Rad, J. Antiulcer agents: From plant extracts to phytochemicals in healing promotion. Molecules 2018, 23, 1751. [CrossRef] [PubMed]

12. Sharifi-Rad, J.; Salehi, B.; Stojanović-Radić, Z.Z.; Fokou, P.V.T.; Sharifi-Rad, M.; Mahady, G.B.; Sharifi-Rad, M.; Masjedi, M.-R.; Lawal, T.O.; Ayatollahi, S.A. Medicinal plants used in the treatment of tuberculosis-ethnobotanical and ethnopharmacological approaches. Biotechnol. Adv. 2017, in press. [CrossRef] [PubMed]

13. Sharifi-Rad, J.; Salehi, B.; Varoni, E.M.; Sharopov, F.; Yousaf, Z.; Ayatollahi, S.A.; Kobarfard, F.; Sharifi-Rad, M.; Afdjei, M.H.; Sharifi-Rad, M.; et al. Plants of the Melaleuca genus as antimicrobial agents: From farm to pharmacy. Phytother. Res. 2017, 31, 1475-1494. [CrossRef] [PubMed]

14. Sahraie-Rad, M.; Izadyari, A.; Rakizadeh, S.; Sharifi-Rad, J. Preparation of strong antidandruff shampoo using medicinal plant extracts: A clinical trial and chronic dandruff treatment. Jundishapur J. Nat. Pharm. Prod. 2015, 10, e21517. [CrossRef]

15. Sharifi-Rad, M.; Roberts, T.H.; Matthews, K.R.; Bezerra, C.F.; Morais-Braga, M.F.B.; Coutinho, H.D.; Sharopov, F.; Salehi, B.; Yousaf, Z.; Sharifi-Rad, M. Ethnobotany of the genus Taraxacum-Phytochemicals and antimicrobial activity. Phytother. Res. 2018. [CrossRef] [PubMed] 
16. Mishra, A.P.; Sharifi-Rad, M.; Shariati, M.A.; Mabkhot, Y.N.; Al-Showiman, S.S.; Rauf, A.; Salehi, B.; Župunski, M.; Sharifi-Rad, M.; Gusain, P. Bioactive compounds and health benefits of edible Rumex species-a review. Cell. Mol. Biol. 2018, 64, 27-34. [CrossRef]

17. Mishra, A.P.; Saklani, S.; Sharifi-Rad, M.; Iriti, M.; Salehi, B.; Maurya, V.K.; Rauf, A.; Milella, L.; Rajabi, S.; Baghalpour, N. Antibacterial potential of Saussurea obvallata petroleum ether extract: A spiritually revered medicinal plant. Cell. Mol. Biol. (Noisy-le-Grand France) 2018, 64, 65-70. [CrossRef]

18. Monjazeb, M.L.; Abdolshahi, A.; Hedayati, S.; Sharifi-Rad, M.; Iriti, M.; Salehi, B.; Sharifi-Rad, J. Pullulan gum production from low-quality fig syrup using Aureobasidium pullulans. Cell. Mol. Biol. (Noisy-le-Grand France) 2018, 64, 22-26. [CrossRef]

19. Salehi, B.; Valussi, M.; Jugran, A.K.; Martorell, M.; Ramírez-Alarcón, K.; Stojanović-Radić, Z.Z.; Antolak, H.; Kregiel, D.; Mileski, K.S.; Sharifi-Rad, M.; et al. Nepeta species: From farm to food applications and phytotherapy. Trends Food Sci. Technol. 2018, 80, 104-122. [CrossRef]

20. Lawrence, B.M. Mint: The Genus Mentha; CRC Press: Boca Raton, FL, USA, 2006.

21. Mamadalieva, N.Z.; Akramov, D.K.; Ovidi, E.; Tiezzi, A.; Nahar, L.; Azimova, S.S.; Sarker, S.D. Aromatic medicinal plants of the Lamiaceae family from Uzbekistan: Ethnopharmacology, essential oils composition, and biological activities. Medicines 2017, 4, 8. [CrossRef] [PubMed]

22. Brahmi, F.; Khodir, M.; Mohamed, C.; Pierre, D. Chemical composition and biological activities of Mentha species. In Aromatic and Medicinal Plants_Back to Nature; InTech: London, England, 2017; pp. 47-80.

23. Tucker, A.O. Mentha: Economic uses. In Mint: The Genus Mentha; Lawrence, B.M., Ed.; CRC Press, Taylor \& Francis Group: Boca Raton, FL, USA, 2007; pp. 519-522.

24. Taneja, S.C.; Chandra, S. Mint. In Handbook of Herbs and Spices; Woodhead Publishing Limited: Sawston, UK, 2012; pp. 366-387.

25. Prakash, O.; Chandra, M.; Pant, A.K.; Rawat, D.S. Mint (Mentha spicata L.) Oils; Elsevier: Amsterdam, The Netherlands, 2016.

26. Patra, N.K.; Kumar, B. Spearmint; Woodhead Publishing Limited: Sawston, UK, 2012.

27. Shaikh, S.; Yaacob, H.B.; Rahim, Z.H.A. Prospective role in treatment of major illnesses and potential benefiits as a safe insecticide and natural food presertive of mint (Mentha spp.): A review. Asian J. Biomed. Pharm. Sci. 2014, 4, 1-12. [CrossRef]

28. Kumar, P.; Mishra, S.; Malik, A.; Satya, S. Insecticidal properties of Mentha species: A review. Ind. Crops Prod. 2011, 34, 802-817. [CrossRef]

29. Murad, H.A.; Abdallah, H.M.; Ali, S.S. Mentha longifolia protects against acetic-acid induced colitis in rats. J. Ethnopharmacol. 2016, 190, 354-361. [CrossRef] [PubMed]

30. Mahboubi, M. Mentha spicata as natural analgesia for treatment of pain in osteoarthritis patients. Complement. Ther. Clin. Pract. 2017, 26, 1-4. [CrossRef] [PubMed]

31. Morcia, C.; Tumino, G.; Ghizzoni, R.; Terzi, V. Carvone (Mentha spicata L.) oil. In Essential Oils in Food Preservation, Flavor and Safety; Elsevier: Amsterdam, The Netherlands, 2016; pp. 309-316.

32. Abdelli, M.; Moghrani, H.; Aboun, A.; Maachi, R. Algerian Mentha pulegium L. Leaves essential oil: Chemical composition, antimicrobial, insecticidal and antioxidant activities. Ind. Crops Prod. 2016, 94, 197-205. [CrossRef]

33. Mahdavikia, F.; Saharkhiz, M.J. Phytotoxic activity of essential oil and water extract of peppermint (Mentha $\times$ piperita L. Cv. Mitcham). J. Appl. Res. Med. Aromat. Plants 2015, 2, 146-153. [CrossRef]

34. Mahdavikia, F.; Saharkhiz, M.J.; Karami, A. Defensive response of radish seedlings to the oxidative stress arising from phenolic compounds in the extract of peppermint (Mentha $\times$ piperita L.). Sci. Horticult. 2017, 214, 133-140. [CrossRef]

35. Hanafy, D.M.; Prenzler, P.D.; Burrows, G.E.; Ryan, D.; Nielsen, S.; El Sawi, S.A.; El Alfy, T.S.; Abdelrahman, E.H.; Obied, H.K. Biophenols of mints: Antioxidant, acetylcholinesterase, butyrylcholinesterase and histone deacetylase inhibition activities targeting alzheimer's disease treatment. J. Funct. Foods 2017, 33, 345-362. [CrossRef]

36. Ferhat, M.; Erol, E.; Beladjila, K.A.; Çetintaş, Y.; Duru, M.E.; Öztürk, M.; Kabouche, A.; Kabouche, Z. Antioxidant, anticholinesterase and antibacterial activities of Stachys guyoniana and Mentha aquatica. Pharm. Biol. 2017, 55, 324-329. [CrossRef] [PubMed] 
37. Arantes, S.; Piçarra, A.; Candeias, F.; Caldeira, A.T.; Martins, M.R.; Teixeira, D. Antioxidant activity and cholinesterase inhibition studies of four flavouring herbs from Alentejo. Nat. Prod. Res. 2017, 31, 2183-2187. [CrossRef] [PubMed]

38. Orhan, I.; Kartal, M.; Kan, Y.; Sener, B. Activity of essential oils and individual components against acetyland butyrylcholinesterase. Z. Naturforsch. C 2008, 63, 547-553. [CrossRef] [PubMed]

39. Chambers, H.L.; Hummer, K.E. Chromosome counts in the Mentha collection at the usda: Ars national clonal germplasm repository. Taxon 1994, 43, 423-432. [CrossRef]

40. Duriyaprapan, S.; Britten, E.; Basford, K. The effect of temperature on growth, oil yield and oil quality of japanese mint. Ann. Bot. 1986, 58, 729-736. [CrossRef]

41. Ringuelet, J.A.; Cerimele, E.L.; Henning, C.P.; Rí, M.S.; Urrutia, M.I. Propagation methods and leaf yield in peppermint (Mentha $\times$ piperita L.). J. Herbs Spices Med. Plants 2003, 10, 55-60. [CrossRef]

42. Shukla, P.; Haseeb, A.; Srivastava, N. The relation between soil ph and the reproduction/damage potential of pratylenchus thornei on growth and oil yield of Mentha spicata. Nematol. Mediterr. 1997, 25, 25-29.

43. Galambosi, B. Mauste-ja Rohdosyrttien Luonnonmukainen Viljely; Painatuskeskus: Helsinki, Finland, 1995; p. 234.

44. Putievsky, E. Cultivation and Processing of Medicinal Plants; John Wiley \& Sons, Inc.: New York, NY, USA, 1992.

45. El-Keltawi, N.E.; Croteau, R. Single-node cuttings as a new method of mint propagation. Sci. Horticult. 1986, 29, 101-105. [CrossRef]

46. Farooqi, A.A. Sreeramu, B. Cultivation of Medicinal and Aromatic Crops; Orient BlackSwan/Universities Press: New Delhi, India, 2004.

47. Aflatuni, A. The Comparative Study of Mint Species Grown in Northern Finland; Agricultural Research Centre of Finland: Jokioinen, Finland, 1999; Volume 66, pp. 74-81.

48. Atanasov, Z.; Slavov, S.; Decheva, R.; Koseva, D.; Gargova, N. Application of Single and Compound Mineral Fertilizers to Peppermint [Nitrogen, Phosphate, Potassium]. Available online: http:/ /agris.fao.org/agrissearch/search.do?recordID=BG8000075 (accessed on 2 September 2018).

49. Clark, R.J.; Menary, R.C. The effect of irrigation and nitrogen on the yield and composition of peppermint oil (Mentha piperita L.). Aust. J. Agric. Res. 1980, 31, 489-498. [CrossRef]

50. Singh, V.; Chatterjee, B.; Singh, D. Response of mint species to nitrogen fertilization. J. Agric. Sci. 1989, 113, 267-271. [CrossRef]

51. Helsel, Z.R. Energy and alternatives for fertilizer and pesticide use. Energy Farm Prod. 1992, 6, 177-201.

52. Okwany, R.O.; Peters, T.R.; Ringer, K.L.; Walsh, D.B.; Rubio, M. Impact of sustained deficit irrigation on spearmint (Mentha spicata L.) biomass production, oil yield, and oil quality. Irrig. Sci. 2012, 30, 213-219. [CrossRef]

53. Charles, D.J.; Joly, R.J.; Simon, J.E. Effects of osmotic stress on the essential oil content and composition of peppermint. Phytochemistry 1990, 29, 2837-2840. [CrossRef]

54. Mitchell, A.; Yang, C. Alternating furrow irrigation of peppermint (Mentha piperita). HortScience 1998, 33, 266-269.

55. Delfine, S.; Loreto, F.; Pinelli, P.; Tognetti, R.; Alvino, A. Isoprenoids content and photosynthetic limitations in rosemary and spearmint plants under water stress. Agric. Ecosyst. Environ. 2005, 106, 243-252. [CrossRef]

56. Okwany, R.O.; Peters, R.; Ringer, K. Effect of sustained deficit irrigation on hay and oil yield of native spearmint (Mentha spicata). In Proceedings of the 5th International Conference on Irrigation and Drainage for Food, Energy and the Environment, Salt Lake City, UT, USA, 3-6 November 2009; pp. 239-252.

57. Croteau, R. Effect of irrigation method on essential oil yield and rate of oil evaporation in mint grown under controlled conditions [Mentha piperita, Mentha spicata]. HortScience 1977, 12, 563-565.

58. Riachi, L.G.; de Maria, C.A.B. Peppermint antioxidants revisited. Food Chem. 2015, 176, 72-81. [CrossRef] [PubMed]

59. Tiwari, P. Recent advances and challenges in trichome research and essential oil biosynthesis in Mentha arvensis L. Ind. Crops Prod. 2016, 82, 141-148. [CrossRef]

60. Croteau, R.B.; Davis, E.M.; Ringer, K.L.; Wildung, M.R. (-)-menthol biosynthesis and molecular genetics. Naturwissenschaften 2005, 92, 562-577. [CrossRef] [PubMed]

61. Kalra, A.; Singh, H.; Pandey, R.; Samad, A.; Patra, N.; Kumar, S. Diseases in mint: Causal organisms, distribution, and control measures. J. Herbs Spices Med. Plants 2005, 11, 71-91. [CrossRef] 
62. Ganguly, D.; Pandotra, V. Some of the commonly occurring diseases of important medicinal and aromatic plants in jammu and kashmir. Ind. Phytopathol. 1962, 15, 50-54.

63. Johnson, D.A.; Santo, G.S. Development of wilt in mint in response to infection by two pathotypes of verticillium dahliae and co-infection by pratylenchus penetrans. Plant Dis. 2001, 85, 1189-1192. [CrossRef]

64. Singh, A.; Singh, H.B. Control of collar rot in mint (Mentha spp.) caused by sclerotium rolfsii using biological means. Curr. Sci. 2004, 87, 362-366.

65. Juronis, V.; Snieskiene, V. Diversity of phytophagous and pathogens and their damage to mints (Mentha). Medicina (Kaunas Lithuania) 2004, 40, 779-782. [PubMed]

66. Zimowska, B. Fungi colonizing and damaging different parts of peppermint (Mentha piperita L.) cultivated in south-eastern poland. Herb. Pol. 2007, 53, 97-105.

67. Dung, J.K.; Schroeder, B.K.; Johnson, D.A. Evaluation of verticillium wilt resistance in Mentha arvensis and M. Longifolia genotypes. Plant Dis. 2010, 94, 1255-1260. [CrossRef]

68. Esfahani, M.N.; Monazzah, M. Identification and assessment of fungal diseases of major medicinal plants. J. Ornament. Horticult. Plants 2011, 1, 137-145.

69. Haseeb, A.; Pandey, R. Observations on meloidogyne spp. Affecting japanese mint: New host records. Nematropica 1989, 19, 93-97.

70. Faulkner, L. Pathogenicity and population dynamics of paratylenchus hamatus on Mentha spp. Phytopathology 1964, 54, 344-348.

71. Maia, N.; Malavolta, J.; Carvalho, R.; Fancelli, M.; Carmello, Q. Occurrence of Pseudomonas cichorii in Mentha arvensis. Summa Phytopathol. 1996, 22, 185-188.

72. Zhou, X.; Yuan, X.; Wang, S. Two new virus diseases found on spearmint. Acta Agric. Shanghai 1990, 6, 45-52.

73. Samad, A.; Zaim, M.; Ajayakumar, P. An outbreak of mosaic disease on mint (Mentha cardiaca baker) in india. Indian J. Plant Pathol. 1994, 12, 1-4.

74. Pandey, R.; Haseeb, A.; Hussain, A. Distribution, pathogencity and management of meloidogyne incognita on Mentha arvensis. Afro-Asian J. Nematol. 1992, 2, 27-34.

75. Shukla, R.; Alam, M.; Kumar, B.; Singh, V. Multiple disease resistance in menthol mint genotypes. Curr. Sci. 2008, 95, 836-838.

76. Shah, S.; Gupta, L. Response of Mentha species to different harvesting intervals. Prog. Hort. 1989, 21, 148-150.

77. Clark, R.J.; Menary, R.C. The effect of two harvests per year on the yield and composition of tasmanian peppermint oil (Mentha piperita L.). J. Sci. Food Agric. 1984, 35, 1191-1195. [CrossRef]

78. Ram, M.; Kumar, S. Optimization of interplant space and harvesting time for high essential oil yield in different varieties of mint Mentha arvensis. J. Med. Aromat. Plant Sci. 1999, 21, 38-45.

79. Chopra, I.C. Cultivation of Medicinal Plants in Jammu and Kashmir; Indian Council of Agricultural Research: New Delhi, India, 1962.

80. Das, K. Medicinal Plants_-Their Importance in Pharmaceutical Sciences; Kalyani Publishers: New Delhi, India, 2010.

81. Sharifi-Rad, M.; Varoni, E.M.; Iriti, M.; Martorell, M.; Setzer, W.N.; del Mar Contreras, M.; Salehi, B.; Soltani-Nejad, A.; Rajabi, S.; Tajbakhsh, M.; et al. Carvacrol and human health: A comprehensive review. Phytother. Res. 2018. [CrossRef] [PubMed]

82. Croteau, R. Metabolism of monoterpenes in mint (Mentha) species. Planta Med. 1991, 57, 10-14. [CrossRef] [PubMed]

83. De Sousa Guedes, J.P.; da Costa Medeiros, J.A.; de Silva, R.S.d.S.; de Sousa, J.M.B.; da Conceição, M.L.; de Souza, E.L. The efficacy of Mentha arvensis L. And M. Piperita L. Essential oils in reducing pathogenic bacteria and maintaining quality characteristics in cashew, guava, mango, and pineapple juices. Int. J. Food Microbiol. 2016, 238, 183-192. [CrossRef] [PubMed]

84. El-Kashoury, E.-S.A.; El-Askary, H.I.; Kandil, Z.A.; Salem, M.A. Chemical composition of the essential oil and botanical study of the flowers of Mentha suaveolens. Pharm. Biol. 2014, 52, 688-697. [CrossRef] [PubMed]

85. Rodrigues, L.; Duarte, A.; Figueiredo, A.C.; Brito, L.; Teixeira, G.; Moldão, M.; Monteiro, A. Chemical composition and antibacterial activity of the essential oils from the medicinal plant Mentha cervina L. Grown in portugal. Med. Chem. Res. 2012, 21, 3485-3490. [CrossRef]

86. Sharopov, F.S.; Sulaimonova, V.A.; Setzer, W.N. Essential oil composition of Mentha longifolia from wild populations growing in Tajikistan. J. Med. Active Plants 2012, 1, 76-84. 
87. Sartoratto, A.; Machado, A.L.M.; Delarmelina, C.; Figueira, G.M.; Duarte, M.C.T.; Rehder, V.L.G. Composition and antimicrobial activity of essential oils from aromatic plants used in Brazil. Braz. J. Microbiol. 2004, 35, 275-280. [CrossRef]

88. Rossi, P.-G.; Berti, L.; Panighi, J.; Luciani, A.; Maury, J.; Muselli, A.; Serra, D.d.R.; Gonny, M.; Bolla, J.-M. Antibacterial action of essential oils from Corsica. J. Essent. Oil Res. 2007, 19, 176-182. [CrossRef]

89. Getahun, Z.; Asres, K.; Mazumder, A.; Bucar, F. Essential oil composition, antibacterial and antioxidant activities of Mentha aquatica growing in Ethiopia. Ethiopian Pharm. J. 2008, 26, 9-16. [CrossRef]

90. Bădulescu, L.; Săvulescu, E.; Delian, E.; Dobrescu, A.; Georgescu, M.; Badea, M.; Ciocârlan, V. The secretory structures and volatile oil composition of Mentha aquatica L. From danube delta. Lucrări Știintifice-Universitatea de Științe Agronomice și Medicină Veterinară București. Seria B Horticultură 2010, 54, 625-628.

91. Sutour, S.; Tomi, F.; Bradesi, P.; Casanova, J. Chemical composition of the essential oil from corsican Mentha aquatica-Combined analysis by GC (RI), GC-MS and 13c nmr spectroscopy. Nat. Prod. Commun. 2011, 6, 1479-1482. [PubMed]

92. Dhifi, W.; Litaiem, M.; Jelali, N.; Hamdi, N.; Mnif, W. Identification of a new chemotye of the plant Mentha aquatica grown in Tunisia: Chemical composition, antioxidant and biological activities of its essential oil. J. Essent. Oil Bear. Plants 2011, 14, 320-328. [CrossRef]

93. Başer, K.; Kürkçüoğlu, M.; Demirci, B.; Özek, T.; Tarımcılar, G. Essential oils of Mentha species from marmara region of Turkey. J. Essent. Oil Res. 2012, 24, 265-272. [CrossRef]

94. Boz, I.; Zamfirache, M.-M.; Burzo, I. Chemical composition of essential oils from Mentha aquatica L. At different moments of the ontogenetic cycle. J. Med. Plants Res. 2013, 7, 470-473.

95. Do Ngoc Dai, T.D.T.; Emmanuel, E.E.; Oladimeji, O.; Abdulkabir, I.A.O. Study on essential oil of Mentha aquatica L. From Vietnam. Am. J. Essent. Oils Nat. Prod. 2015, 2, 12-16.

96. de Sousa Barros, A.; de Morais, S.M.; Ferreira, P.A.T.; Vieira, Í.G.P.; Craveiro, A.A.; dos Santos Fontenelle, R.O.; de Menezes, J.E.S.A.; da Silva, F.W.F.; de Sousa, H.A. Chemical composition and functional properties of essential oils from Mentha species. Ind. Crops Prod. 2015, 76, 557-564. [CrossRef]

97. Souza, M.A.; Araújo, O.J.; Brito, D.M.; Fernandes, M.S.; Castro, R.N.; Souza, S.R. Chemical composition of the essential oil and nitrogen metabolism of menthol mint under different phosphorus levels. Am. J. Plant Sci. 2014, 5, 2312. [CrossRef]

98. Yu, X.; Liang, C.; Chen, J.; Qi, X.; Liu, Y.; Li, W. The effects of salinity stress on morphological characteristics, mineral nutrient accumulation and essential oil yield and composition in Mentha canadensis L. Sci. Horticult. 2015, 197, 579-583. [CrossRef]

99. Ji, H.; Li, Y.C.; Wen, Z.y.; Li, X.H.; Zhang, H.X.; Li, H.T. Gc-ms analysis of nematicidal essential oil of Mentha canadensis aerial parts against heterodera avenae and meloidogyne incognita. J. Essent. Oil Bear. Plants 2016, 19, 2056-2064. [CrossRef]

100. Dwivedy, A.K.; Prakash, B.; Chanotiya, C.S.; Bisht, D.; Dubey, N.K. Chemically characterized Mentha cardiaca L. Essential oil as plant based preservative based on efficacy against biodeteriorating fungi of dry fruits, aflatoxin secretion, lipid peroxidation and safety profile assessment. Food Chem. Toxicol. 2017, 106, 175-184. [CrossRef] [PubMed]

101. Gonçalves, M.; Vicente, A.; Cavaleiro, C.; Salgueiro, L. Composition and antifungal activity of the essential oil of Mentha cervina from Portugal. Nat. Prod. Res. 2007, 21, 867-871. [CrossRef] [PubMed]

102. Rodrigues, L.; Monteiro, P.; Póvoa, O.; Teixeira, G.; Moldão, M.; Figueiredo, A.C.; Monteiro, A. Morphology of secretory structures and essential oil composition in Mentha cervina L. from Portugal. Flavour Fragrance J. 2008, 23, 340-347. [CrossRef]

103. Brophy, J.J.; Goldsack, R.J.; Lawrence, B.M.; Forster, P.I. Essential oil of Mentha diemenica (Lamiaceae). J. Essent. Oil Res. 1996, 8, 179-181. [CrossRef]

104. Okut, N.; Yagmur, M.; Selcuk, N.; Yildirim, B. Chemical composition of essential oil of Mentha longifolia L. Subsp. Longifolia growing wild. Pak. J. Bot. 2017, 49, 525-529.

105. Golparvar, A.R.; Hadipanah, A.; Gheisari, M.M.; Salehi, S.; Khaliliazar, R.; Ghasemi, O. Comparative analysis of chemical composition of Mentha longifolia (L.) huds. J. Herb. Drugs (Int. J. Med. Herbs) 2017, 7, $235-241$.

106. Salman, M.; Abdel-Hameed, E.; Bazaid, S.; Dabi, M.M. Chemical composition for hydrodistillation essential oil of Mentha longifolia by gas chromatography-mass spectrometry from north regions in kingdom of Saudi Arabia. Pharm. Chem. 2015, 7, 34-40. 
107. Niksic, H.; Besovic, E.K.; Makarevic, E.; Duric, K. Chemical composition, antimicrobial and antioxidant properties of Mentha longifolia (L.) Huds. Essential oil. J. Health Sci. 2012, 2, 192-200. [CrossRef]

108. Mkaddem, M.; Bouajila, J.; Ennajar, M.; Lebrihi, A.; Mathieu, F.; Romdhane, M. Chemical composition and antimicrobial and antioxidant activities of Mentha (longifolia L. and viridis) essential oils. J. Food Sci. 2009, 74, 358-363. [CrossRef] [PubMed]

109. Ouakouak, H.; Chohra, M.; Denane, M. Chemical composition, antioxidant activities of the essential oil of Mentha pulegium L., south east of Algeria. Int. Lett. Nat. Sci. 2015, 39, 49-55. [CrossRef]

110. Bouyahya, A.; Et-Touys, A.; Bakri, Y.; Talbaui, A.; Fellah, H.; Abrini, J.; Dakka, N. Chemical composition of Mentha pulegium and Rosmarinus officinalis essential oils and their antileishmanial, antibacterial and antioxidant activities. Microbial. Pathog. 2017, 111, 41-49. [CrossRef] [PubMed]

111. Benayad, N.; Ebrahim, W.; Hakiki, A.; Mosaddak, M. Chemical characterization and insecticidal evaluation of the essential oil of Mentha suaveolens L. and Mentha pulegium L. Growing in Morocco. Food Ind. Bacau 2012, 13, 27-32.

112. Lorenzo, D.; Paz, D.; Dellacassa, E.; Davies, P.; Vila, R.; Cañigueral, S. Essential oils of Mentha pulegium and Mentha rotundifolia from Uruguay. Braz. Arch. Biol. Technol. 2002, 45, 519-524. [CrossRef]

113. Boukhebti, H.; Chaker, A.N.; Belhadj, H.; Sahli, F.; Ramdhani, M.; Laouer, H.; Harzallah, D. Chemical composition and antibacterial activity of Mentha pulegium L. and Mentha spicata L. Essential oils. Pharm. Lett. 2011, 3, 267-275.

114. Teixeira, B.; Marques, A.; Ramos, C.; Batista, I.; Serrano, C.; Matos, O.; Neng, N.R.; Nogueira, J.M.; Saraiva, J.A.; Nunes, M.L. European pennyroyal (Mentha pulegium) from portugal: Chemical composition of essential oil and antioxidant and antimicrobial properties of extracts and essential oil. Ind. Crops Prod. 2012, 36, 81-87. [CrossRef]

115. El Asbahani, A.; Jilale, A.; Voisin, S.N.; Aït Addi, E.h.; Casabianca, H.; El Mousadik, A.; Hartmann, D.J.; Renaud, F.N. Chemical composition and antimicrobial activity of nine essential oils obtained by steam distillation of plants from the souss-massa region (Morocco). J. Essent. Oil Res. 2015, 27, 34-44. [CrossRef]

116. Govindarajan, M.; Sivakumar, R.; Rajeswari, M.; Yogalakshmi, K. Chemical composition and larvicidal activity of essential oil from Mentha spicata (Linn.) against three mosquito species. Parasitol. Res. 2012, 110, 2023-2032. [CrossRef] [PubMed]

117. Teixeira, M.L.; Cardoso, M.d.G.; Figueiredo, A.C.S.; Moraes, J.C.; Assis, F.A.; de Andrade, J.; Nelson, D.L.; de Souza Gomes, M.; de Souza, J.A.; de Albuquerque, L.R.M. Essential oils from Lippia origanoides Kunth. And Mentha spicata L.: Chemical composition, insecticidal and antioxidant activities. Am. J. Plant Sci. 2014, 5, 1181. [CrossRef]

118. Snoussi, M.; Noumi, E.; Trabelsi, N.; Flamini, G.; Papetti, A.; De Feo, V. Mentha spicata essential oil: Chemical composition, antioxidant and antibacterial activities against planktonic and biofilm cultures of Vibrio spp. strains. Molecules 2015, 20, 14402-14424. [CrossRef] [PubMed]

119. Diop, S.M.; Guèye, M.T.; Ndiaye, I.; Ndiaye, E.H.B.; Diop, M.B.; Heuskin, S.; Fauconnier, M.-L.; Lognay, G. Chemical characterization of essential oils of mints from Senegal. Nat. Prod. Commun. 2016, 11, 1-2.

120. Soković, M.D.; Vukojević, J.; Marin, P.D.; Brkić, D.D.; Vajs, V.; Van Griensven, L.J. Chemical composition of essential oilsof Thymus and Mentha species and their antifungal activities. Molecules 2009, 14, 238-249. [CrossRef] [PubMed]

121. Koliopoulos, G.; Pitarokili, D.; Kioulos, E.; Michaelakis, A.; Tzakou, O. Chemical composition and larvicidal evaluation of Mentha, Salvia, and Melissa essential oils against the west nile virus mosquito culex pipiens. Parasitol. Res. 2010, 107, 327-335. [CrossRef] [PubMed]

122. Chauhan, R.; Kaul, M.; Shahi, A.; Kumar, A.; Ram, G.; Tawa, A. Chemical composition of essential oils in Mentha spicata L. Accession [iiim (j) 26] from north-west himalayan region, India. Ind. Crops Prod. 2009, 29, 654-656. [CrossRef]

123. Tomei, P.E.; Uncini Manganelli, R.E.; Flamini, G.; Cioni, P.L.; Morelli, I. Composition of the essential oil of Mentha microphylla from the gennargentu mountains (Sardinia, Italy). J. Agric. Food Chem. 2003, 51, 3614-3617. [CrossRef] [PubMed]

124. El-Kashoury, E.-S.A.; El-Askary, H.I.; Kandil, Z.A.; Salem, M.A.; Sleem, A.A. Chemical composition and biological activities of the essential oil of Mentha suaveolens ehrh. Z. Naturforsch. C 2012, 67, 571-579. [CrossRef] 
125. Oumzil, H.; Ghoulami, S.; Rhajaoui, M.; Ilidrissi, A.; Fkih-Tetouani, S.; Faid, M.; Benjouad, A. Antibacterial and antifungal activity of essential oils of Mentha suaveolens. Phytother. Res. 2002, 16, 727-731. [CrossRef] [PubMed]

126. Wang, J.; Li, R.; Tan, J.; Jiang, Z.-T. Chemical composition of essential oil of grapefruit mint (Mentha suaveolens $\times$ piperita) from China. J. Essent. Oil Bearing Plants 2016, 19, 1047-1050. [CrossRef]

127. Tyagi, A.K.; Malik, A. Antimicrobial potential and chemical composition of Mentha piperita oil in liquid and vapour phase against food spoiling microorganisms. Food Control 2011, 22, 1707-1714. [CrossRef]

128. Derwich, E.; Benziane, Z.; Taouil, R.; Senhaji, O.; Touzani, M. Aromatic plants of Morocco: GC/MS analysis of the essential oils of leaves of Mentha piperita. Adv. Environ. Biol. 2010, 4, 80-86.

129. Moghaddam, M.; Pourbaige, M.; Tabar, H.K.; Farhadi, N.; Hosseini, S.M.A. Composition and antifungal activity of peppermint (Mentha piperita) essential oil from iran. J. Essent. Oil Bear. Plants 2013, 16, 506-512. [CrossRef]

130. Taherpour, A.A.; Khaef, S.; Yari, A.; Nikeafshar, S.; Fathi, M.; Ghambari, S. Chemical composition analysis of the essential oil of Mentha piperita L. From kermanshah, Iran by hydrodistillation and HS/SPME methods. J. Anal. Sci. Technol. 2017, 8, 11. [CrossRef]

131. Sun, Z.; Wang, H.; Wang, J.; Zhou, L.; Yang, P. Chemical composition and anti-inflammatory, cytotoxic and antioxidant activities of essential oil from leaves of Mentha piperita grown in China. PLoS ONE 2014, 9, e114767. [CrossRef] [PubMed]

132. Tsai, M.-L.; Wu, C.-T.; Lin, T.-F.; Lin, W.-C.; Huang, Y.-C.; Yang, C.-H. Chemical composition and biological properties of essential oils of two mint species. Trop. J. Pharm. Res. 2013, 12, 577-582. [CrossRef]

133. Reddy, D.N.; Al-Rajab, A.J.; Sharma, M.; Moses, M.M.; Reddy, G.R.; Albratty, M. Chemical constituents, in vitro antibacterial and antifungal activity of Mentha $\times$ piperita L. (peppermint) essential oils. J. King Saud Univ.-Sci. 2017. [CrossRef]

134. Satmi, F.R.S.; Hossain, M.A. In vitro antimicrobial potential of crude extracts and chemical compositions of essential oils of leaves of Mentha piperita L. native to the sultanate of Oman. Pac. Sci. Rev. A Nat. Sci. Eng. 2016, 18, 103-106. [CrossRef]

135. Hussain, A.I.; Anwar, F.; Nigam, P.S.; Ashraf, M.; Gilani, A.H. Seasonal variation in content, chemical composition and antimicrobial and cytotoxic activities of essential oils from four Mentha species. J. Sci. Food Agric. 2010, 90, 1827-1836. [CrossRef] [PubMed]

136. Chowdhury, J.U.; Nandi, N.C.; Uddin, M.; Rahman, M. Chemical constituents of essential oils from two types of spearmint (Mentha spicata L. and M. cardiaca L.) introduced in Bangladesh. Bangladesh J. Sci. Ind. Res. 2007, 42, 79-82. [CrossRef]

137. Rezaei, M.B.; Jaymand, K.; Jamzad, Z. Chemical constituents of Mentha longifolia (L.) Hudson var. Chlorodictya rech. F. From three different localities. Pajouhesh-Va-Sazandegi 2000, 13, 60-63.

138. Mimica-Dukić, N.; Božin, B.; Soković, M.; Mihajlović, B.; Matavulj, M. Antimicrobial and antioxidant activities of three Mentha species essential oils. Planta Med. 2003, 69, 413-419. [PubMed]

139. Viljoen, A.M.; Petkar, S.; Van Vuuren, S.F.; Figueiredo, A.C.; Pedro, L.G.; Barroso, J.G. The chemo-geographical variation in essential oil composition and the antimicrobial properties of "wild mint" - Mentha longifolia subsp. Polyadena (Lamiaceae) in southern Africa. J. Essent. Oil Res. 2006, 18, 60-65.

140. Gulluce, M.; Sahin, F.; Sokmen, M.; Ozer, H.; Daferera, D.; Sokmen, A.; Polissiou, M.; Adiguzel, A.; Ozkan, H. Antimicrobial and antioxidant properties of the essential oils and methanol extract from Mentha longifolia L. ssp. Longifolia. Food Chem. 2007, 103, 1449-1456. [CrossRef]

141. Hafedh, H.; Fethi, B.A.; Mejdi, S.; Emira, N.; Amina, B. Effect of Mentha longifolia L. ssp longifolia essential oil on the morphology of four pathogenic bacteria visualized by atomic force microscopy. Afr. J. Microbiol. Res. 2010, 4, 1122-1127.

142. Džamić, A.M.; Soković, M.D.; Ristić, M.S.; Novaković, M.; Grujić-Jovanović, S.; Tešević, V.; Marin, P.D. Antifungal and antioxidant activity of Mentha longifolia (L.) Hudson (Lamiaceae) essential oil. Botanica Serbica 2010, 34, 57-61.

143. Božović, M.; Pirolli, A.; Ragno, R. Mentha suaveolens Ehrh.(Lamiaceae) essential oil and its main constituent piperitenone oxide: Biological activities and chemistry. Molecules 2015, 20, 8605-8633. [CrossRef] [PubMed]

144. Mimica-Dukic, N.; Bozin, B. Mentha L. Species (Lamiaceae) as promising sources of bioactive secondary metabolites. Curr. Pharm. Des. 2008, 14, 3141-3150. [CrossRef] [PubMed] 
145. Tang, K.S.C.; Konczak, I.; Zhao, J. Identification and quantification of phenolics in australian native mint (Mentha australis r. Br.). Food Chem. 2016, 192, 698-705. [CrossRef] [PubMed]

146. Pei, X.; Li, N.; Guo, Y.; Liu, X.; Yan, L.; Li, Y.; Yang, S.; Hu, J.; Zhu, J.; Yang, D. Microbiological food safety surveillance in China. Int. J. Environ. Res. Public Health 2015, 12, 10662-10670. [CrossRef] [PubMed]

147. World Health Organization. Who's First Ever Global Estimates of Foodborne Diseases Find Children under 5 Account for Almost Third of Deaths; World Health Organization: Geneva, Switzerland, 2015.

148. Hussain, M.A. Food Contamination: Major Challenges of the Future; Multidisciplinary Digital Publishing Institute: Basel, Switzerland, 2016.

149. Abdolshahi, A.; Naybandi-Atashi, S.; Heydari-Majd, M.; Salehi, B.; Kobarfard, F.; Ayatollahi, S.A.; Ata, A.; Tabanelli, G.; Sharifi-Rad, M.; Montanari, C. Antibacterial activity of some Lamiaceae species against Staphylococcus aureus in yoghurt-based drink (Doogh). Cell. Mol. Biol. (Noisy-le-Grand France) 2018, 64, 71-77. [CrossRef]

150. Sancho-Madriz, M.F. Preservation of food. In Encyclopedia of Food Sciences and Nutrition (Second Edition), Caballero, B., Ed.; Academic Press: Oxford, UK, 2003; pp. 4766-4772.

151. Sharifi-Rad, M.; Ozcelik, B.; Altın, G.; Daşkaya-Dikmen, C.; Martorell, M.; Ramírez-Alarcón, K.; Alarcón-Zapata, P.; Morais-Braga, M.F.B.; Carneiro, J.N.P.; Alves Borges Leal, A.L. Salvia spp. plants-from farm to food applications and Phytopharmacotherapy. Trends Food Sci. Technol. 2018. [CrossRef]

152. Raeisi, S.; Sharifi-Rad, M.; Quek, S.Y.; Shabanpour, B.; Sharifi-Rad, J. Evaluation of antioxidant and antimicrobial effects of shallot (Allium ascalonicum L.) fruit and ajwain (Trachyspermum ammi (L.) sprague) seed extracts in semi-fried coated rainbow trout (Oncorhynchus mykiss) fillets for shelf-life extension. LWT-Food Sci. Technol. 2016, 65, 112-121. [CrossRef]

153. Raeisi, S.; Ojagh, S.M.; Sharifi-Rad, M.; Sharifi-Rad, J.; Quek, S.Y. Evaluation of Allium paradoxum (M.B.) G. Don. and Eryngium caucasicum trauve. Extracts on the shelf-life and quality of silver carp (Hypophthalmichthys molitrix) fillets during refrigerated storage. J. Food Saf. 2017, 37, e12321. [CrossRef]

154. Benabdallah, A.; Rahmoune, C.; Boumendjel, M.; Aissi, O.; Messaoud, C. Total phenolic content and antioxidant activity of six wild Mentha species (Lamiaceae) from northeast of Algeria. Asian Pac. J. Trop. Biomed. 2016, 6, 760-766. [CrossRef]

155. Ilboudo, O.; Bonzi, S.; Tapsoba, I.; Somda, I.; Bonzi-Coulibaly, Y.L. In vitro antifungal activity of flavonoid diglycosides of Mentha piperita and their oxime derivatives against two cereals fungi. C. R. Chim. 2016, 19, 857-862. [CrossRef]

156. Sujana, P.; Sridhar, T.M.; Josthna, P.; Naidu, C.V. Antibacterial activity and phytochemical analysis of Mentha piperita L. (peppermint)—An important multipurpose medicinal plant. Am. J. Plant Sci. 2013, 4, 77. [CrossRef]

157. Bayoub, K.; Baibai, T.; Mountassif, D.; Retmane, A.; Soukri, A. Antibacterial activities of the crude ethanol extracts of medicinal plants against listeria monocytogenes and some other pathogenic strains. Afr. J. Biotechnol. 2010, 9, 4251-4258.

158. Bupesh, G.; Amutha, C.; Nandagopal, S.; Ganeshkumar, A.; Sureshkumar, P.; Murali, K. Antibacterial activity of Mentha piperita L. (peppermint) from leaf extracts-a medicinal plant. Acta Agric. Slovenica 2007, 89, 73. [CrossRef]

159. Dhiman, R.; Aggarwal, N.; Aneja, K.R.; Kaur, M. In vitro antimicrobial activity of spices and medicinal herbs against selected microbes associated with juices. Int. J. Microbiol. 2016, 2016, 9015802. [CrossRef] [PubMed]

160. Sandasi, M.; Leonard, C.; Van Vuuren, S.; Viljoen, A. Peppermint (Mentha piperita) inhibits microbial biofilms in vitro. S. Afr. J. Bot. 2011, 77, 80-85. [CrossRef]

161. Antolak, H.; Czyżowska, A.; Kręgiel, D. Anti-adhesion activity of mint (mentha piperita L.) leaves extract against beverage spoilage bacteria Asaia spp. Biotechnol. Food Sci. 2016, 80, 119-127.

162. Panda, P.; Aiko, V.; Mehta, A. Effect of aqueous extracts of Mentha arvensis (mint) and piper betle (betel) on growth and citrinin production from toxigenic Penicillium citrinum. J. Food Sci. Technol. 2015, 52, 3466-3474. [CrossRef] [PubMed]

163. Tassou, C.; Koutsoumanis, K.; Nychas, G.-J. Inhibition of salmonella enteritidis and Staphylococcus aureus in nutrient broth by mint essential oil. Food Res. Int. 2000, 33, 273-280. [CrossRef]

164. Singh, R.; Shushni, M.A.; Belkheir, A. Antibacterial and antioxidant activities of Mentha piperita L. Arabian J. Chem. 2015, 8, 322-328. [CrossRef]

165. Saeed, S.; Tariq, P. Antibacterial activities of Mentha piperita, Pisum sativum and Momordica charantia. Pak. J. Bot. 2005, 37, 997. 
166. Rodriguez-Fragoso, L.; Reyes-Esparza, J.; Burchiel, S.W.; Herrera-Ruiz, D.; Torres, E. Risks and benefits of commonly used herbal medicines in Mexico. Toxicol. Appl. Pharmacol. 2008, 227, 125-135. [CrossRef] [PubMed]

167. Patil, S.R.; Patil, R.S.; Godghate, A. Mentha piperita Linn: Phytochemical, antibacterial and dipterian adulticidal approach. Int. J. Pharm. Pharm. Sci. 2016, 8, 352-355.

168. İşcan, G.; Kirimer, N.; Kürkcüoğlu, M.n.; Başer, H.C.; DEMIrci, F. Antimicrobial screening of Mentha piperita essential oils. J. Agric. Food Chem. 2002, 50, 3943-3946. [CrossRef] [PubMed]

169. Chauret, C. Survival and control of escherichia coli o157: H7 in foods, beverages, soil and water. Virulence 2011, 2, 593-601. [CrossRef] [PubMed]

170. Shahbazi, Y. Chemical composition and in vitro antibacterial activity of Mentha spicata essential oil against common food-borne pathogenic bacteria. J. Pathog. 2015, 2015, 916305. [CrossRef] [PubMed]

171. Aliakbarlu, J.; Sadaghiani, S.K.; Mohammadi, S. Comparative evaluation of antioxidant and anti food-borne bacterial activities of essential oils from some spices commonly consumed in Iran. Food Sci. Biotechnol. 2013, 22, 1487-1493. [CrossRef]

172. Riahi, L.; Elferchichi, M.; Ghazghazi, H.; Jebali, J.; Ziadi, S.; Aouadhi, C.; Chograni, H.; Zaouali, Y.; Zoghlami, N.; Mliki, A. Phytochemistry, antioxidant and antimicrobial activities of the essential oils of Mentha rotundifolia L. In Tunisia. Ind. Crops Prod. 2013, 49, 883-889. [CrossRef]

173. Do Nascimento, E.M.; Rodrigues, F.; Campos, A.; Da Costa, J.G. Phytochemical prospection, toxicity and antimicrobial activity of Mentha arvensis (Labiatae) from northeast of Brazil. J. Young Pharm. 2009, 1, $210-212$.

174. Al-Bayati, F.A. Isolation and identification of antimicrobial compound from Mentha longifolia L. Leaves grown wild in Iraq. Ann. Clin. Microbiol. Antimicrob. 2009, 8, 20. [CrossRef] [PubMed]

175. Chraibi, M.; Fikri-Benbrahim, K.; Ou-yahyia, D.; Balouiri, M.; Farah, A. Radical scavenging and disinfectant effect of essential oil from Moroccan Mentha pulegium. Int. J. Pharm. Pharm. Res. 2016, 8, 116-119. [CrossRef]

176. Aires, A.; Marrinhas, E.; Carvalho, R.; Dias, C.; Saavedra, M.J. Phytochemical composition and antibacterial activity of hydroalcoholic extracts of pterospartum tridentatum and Mentha pulegium against Staphylococcus aureus isolates. BioMed Res. Int. 2016, 2016, 5201879. [CrossRef] [PubMed]

177. Waller, S.B.; Cleff, M.B.; Serra, E.F.; Silva, A.L.; Gomes, A.R.; Mello, J.R.B.; Faria, R.O.; Meireles, M.C.A. Plants from lamiaceae family as source of antifungal molecules in humane and veterinary medicine. Microb. Pathog. 2017, 104, 232-237. [CrossRef] [PubMed]

178. Mahboubi, M.; Haghi, G. Antimicrobial activity and chemical composition of Mentha pulegium L. Essential oil. J. Ethnopharmacol. 2008, 119, 325-327. [CrossRef] [PubMed]

179. Ait-Ouazzou, A.; Lorán, S.; Arakrak, A.; Laglaoui, A.; Rota, C.; Herrera, A.; Pagán, R.; Conchello, P. Evaluation of the chemical composition and antimicrobial activity of Mentha pulegium, juniperus phoenicea, and cyperus longus essential oils from Morocco. Food Res. Int. 2012, 45, 313-319. [CrossRef]

180. Dhifi, W.; Jelali, N.; Mnif, W.; Litaiem, M.; Hamdi, N. Chemical composition of the essential oil of Mentha spicata L. From tunisia and its biological activities. J. Food Biochem. 2013, 37, 362-368. [CrossRef]

181. Soković, M.; Glamočlija, J.; Marin, P.D.; Brkić, D.; van Griensven, L.J. Antibacterial effects of the essential oils of commonly consumed medicinal herbs using an in vitro model. Molecules 2010, 15, 7532-7546. [CrossRef] [PubMed]

182. Ben Arfa, A.; Combes, S.; Preziosi-Belloy, L.; Gontard, N.; Chalier, P. Antimicrobial activity of carvacrol related to its chemical structure. Lett. Appl. Microbiol. 2006, 43, 149-154. [CrossRef] [PubMed]

183. Hyldgaard, M.; Mygind, T.; Meyer, R.L. Essential oils in food preservation: Mode of action, synergies, and interactions with food matrix components. Front. Microbiol. 2012, 3, 1-24. [CrossRef] [PubMed]

184. Ferreira, P.; Cardoso, T.; Ferreira, F.; Fernandes-Ferreira, M.; Piper, P.; Sousa, M.J. Mentha piperita essential oil induces apoptosis in yeast associated with both cytosolic and mitochondrial ros-mediated damage. FEMS Yeast Res. 2014, 14, 1006-1014. [PubMed]

185. Bassolé, I.H.N.; Lamien-Meda, A.; Bayala, B.; Tirogo, S.; Franz, C.; Novak, J.; Nebié, R.C.; Dicko, M.H. Composition and antimicrobial activities of lippia multiflora moldenke, Mentha $\times$ piperita $\mathrm{L}$. and ocimum basilicum L. Essential oils and their major monoterpene alcohols alone and in combination. Molecules 2010, 15, 7825-7839. [CrossRef] [PubMed]

186. Fatoki, O.A.; Onifade, D.A. Use of plant antimicrobials for food preservation. World Acad. Sci. Eng. Technol. Int. J. Biol. Biomol. Agric. Food Biotechnol. Eng. 2013, 7, 1110-1113. 
187. Saharkhiz, M.J.; Motamedi, M.; Zomorodian, K.; Pakshir, K.; Miri, R.; Hemyari, K. Chemical composition, antifungal and antibiofilm activities of the essential oil of Mentha piperita L. ISRN Pharm. 2012, 2012, 718645. [CrossRef] [PubMed]

188. Pandey, A.K.; Kumar, P.; Singh, P.; Tripathi, N.N.; Bajpai, V.K. Essential oils: Sources of antimicrobials and food preservatives. Front. Microbiol. 2017, 7, 2161. [CrossRef] [PubMed]

189. Pandey, A.K.; Sonker, N.; Singh, P. Efficacy of some essential oils against Aspergillus flavus with special reference to Lippia alba oil an inhibitor of fungal proliferation and aflatoxin b1 production in green gram seeds during storage. J. Food Sci. 2016, 81, M928-M934. [CrossRef] [PubMed]

190. Kumar, R.; Dubey, N.K.; Tiwari, O.P.; Tripathi, Y.B.; Sinha, K.K. Evaluation of some essential oils as botanical fungitoxicants for the protection of stored food commodities from fungal infestation. J. Sci. Food Agric. 2007, 87, 1737-1742. [CrossRef]

191. Pandey, A.K.; Tripathi, N. Post-harvest fungal and insect deterioration of Pigeon pea seeds and their management by plant volatiles. J. Ind. Bot. Soc. 2011, 90, 326-331.

192. Beyki, M.; Zhaveh, S.; Khalili, S.T.; Rahmani-Cherati, T.; Abollahi, A.; Bayat, M.; Tabatabaei, M.; Mohsenifar, A. Encapsulation of Mentha piperita essential oils in chitosan-cinnamic acid nanogel with enhanced antimicrobial activity against Aspergillus flavus. Ind. Crops Prod. 2014, 54, 310-319. [CrossRef]

193. Weiss, J.; Loeffler, M.; Terjung, N. The antimicrobial paradox: Why preservatives lose activity in foods. Curr. Opin. Food Sci. 2015, 4, 69-75. [CrossRef]

194. Cava, R.; Nowak, E.; Taboada, A.; Marin-Iniesta, F. Antimicrobial activity of clove and cinnamon essential oils against listeria monocytogenes in pasteurized milk. J. Food Prot. 2007, 70, 2757-2763. [CrossRef] [PubMed]

195. Bakkali, F.; Averbeck, S.; Averbeck, D.; Idaomar, M. Biological effects of essential oils-A review. Food Chem. Toxicol. 2008, 46, 446-475. [CrossRef] [PubMed]

196. Negi, P.S. Plant extracts for the control of bacterial growth: Efficacy, stability and safety issues for food application. Int. J. Food Microbiol. 2012, 156, 7-17. [CrossRef] [PubMed]

197. Bassolé, I.H.N.; Juliani, H.R. Essential oils in combination and their antimicrobial properties. Molecules 2012, 17, 3989-4006. [CrossRef] [PubMed]

198. Klūga, A.; Terentjeva, M.; Kántor, A.; Kluz, M.; Puchalski, C.; Kačániová, M. Antibacterial activity of Melissa officinalis L., Mentha piperita L., Origanum vulgare L. And Malva mauritiana against bacterial microflora isolated from fish. Adv. Res. Life Sci. 2017, 1, 75-80. [CrossRef]

199. Choi, O.; Cho, S.K.; Kim, J.; Park, C.G.; Kim, J. Antibacterial properties and major bioactive components of Mentha piperita essential oils against bacterial fruit blotch of watermelon. Arch. Phytopathol. Plant Prot. 2016, 49, 325-334. [CrossRef]

200. Fazlara, A.; Najafzadeh, H.; Lak, E. The potential application of plant essential oils as natural preservatives against Escherichia coli O157: H7. Pak. J. Biol. Sci. 2008, 11, 2054-2061. [CrossRef] [PubMed]

201. Viji, P.; Binsi, P.K.; Visnuvinayagam, S.; Bindu, J.; Ravishankar, C.N.; Gopal, T.K.S. Efficacy of mint (Mentha arvensis) leaf and citrus (Citrus aurantium) peel extracts as natural preservatives for shelf life extension of chill stored indian mackerel. J. Food Sci. Technol. 2015, 52, 6278-6289. [CrossRef] [PubMed]

202. Karim, G.; Meshgi, M.A.; Ababil, R.K.; Bokaie, S. Antimicrobial effect of Mentha spicata and Mentha pulegium essential oils in two storage temperatures on the survival of Debaryomyces hansenii in Iranian doogh. Appl. Food Biotechnol. 2016, 3, 99-104.

203. Bajaj, S.; Urooj, A.; Prabhasankar, P. Antioxidative properties of mint (Mentha spicata L.) and its application in biscuits. Curr. Res. Nutr. Food Sci. J. 2016, 4, 209-216. [CrossRef]

204. Raeisi, S.; Quek, S.Y.; Ojagh, S.M.; Alishahi, A.R. Effects of cumin (Cuminum cyminum L.) seed and wild mint (Mentha longifolia L.) leaf extracts on the shelf life and quality of rainbow trout (Oncorhynchus mykiss) fillets stored at $4 \mathrm{C} \pm 1$. J. Food Saf. 2016, 36, 271-281. [CrossRef]

205. Sadeghi, E.; Mohammadi, A.; Jamilpanah, M.; Bashiri, M.; Bohlouli, S. Antimicrobial effects of Mentha pulegium essential oil on listeria monocytogenes in iranian white cheese. J. Food Qual. Hazards Control 2016, 3, 20-24.

206. Evrendilek, G.A.; Balasubramaniam, V. Inactivation of listeria monocytogenes and Listeria innocua in yogurt drink applying combination of high pressure processing and mint essential oils. Food Control 2011, 22, 1435-1441. [CrossRef] 
207. Nguyen, P.; Mittal, G. Inactivation of naturally occurring microorganisms in tomato juice using pulsed electric field (pef) with and without antimicrobials. Chem. Eng. Process. Process Intensif. 2007, 46, 360-365. [CrossRef]

208. Karagözlü, N.; Ergönül, B.; Özcan, D. Determination of antimicrobial effect of mint and basil essential oils on survival of E. coli O157: H7 and S. Typhimurium in fresh-cut lettuce and purslane. Food Control 2011, 22, 1851-1855. [CrossRef]

209. Najeeb, A.; Mandal, P.; Pal, U. Efficacy of leaves (drumstick, mint and curry leaves) powder as natural preservatives in restructured chicken block. J. Food Sci. Technol. 2015, 52, 3129-3133. [CrossRef] [PubMed]

210. Tassou, C.; Drosinos, E.; Nychas, G. Effects of essential oil from mint (Mentha piperita) on Salmonella enteritidis and Listeria monocytogenes in model food systems at 4 and 10 C. J. Appl. Microbiol. 1995, 78, 593-600.

211. Ahmad, A.; Khan, A.; Samber, N.; Manzoor, N. Antimicrobial activity of Mentha piperita essential oil in combination with silver ions. Synergy 2014, 1, 92-98. [CrossRef]

212. Smaoui, S.; Hsouna, A.B.; Lahmar, A.; Ennouri, K.; Mtibaa-Chakchouk, A.; Sellem, I.; Najah, S.; Bouaziz, M.; Mellouli, L. Bio-preservative effect of the essential oil of the endemic Mentha piperita used alone and in combination with BacTN635 in stored minced beef meat. Meat Sci. 2016, 117, 196-204. [CrossRef] [PubMed]

(C) 2018 by the authors. Licensee MDPI, Basel, Switzerland. This article is an open access article distributed under the terms and conditions of the Creative Commons Attribution (CC BY) license (http:/ / creativecommons.org/licenses/by/4.0/). 